\title{
The Mechanism of the Phosphoglucomutase Reaction
}

\author{
STUDIES ON RABBIT MUSCLE PHOSPHOGLUCOMUTASE WITH FLUX TECHNIQUES
}

\author{
BY H. G. BRITTON AND J. B. CLARKE \\ Department of Physiology, St Mary's Hospital Medical School, London, W. 2
}

(Received 16 April 1968)

\begin{abstract}
1. The kinetics of phosphoglucomutases from different sources are discussed and it is concluded that on the available evidence there are in all cases three possible mechanisms for the reaction. These are an indirect transfer of phosphate involving the phosphoenzyme (mechanism 1), a direct transfer of phosphate (mechanism 2), and an intermolecular transfer of phosphate from glucose 1,6-diphosphate to the substrate (mechanism 3). Conventional net flux measurements are shown not to differentiate between these mechanisms. 2. Flux equations are developed and it is shown that there are three flux ratios that characterize and distinguish between the mechanisms. 3. To examine these flux ratios induced-transport tests are described with ${ }^{14} \mathrm{C}$ - and ${ }^{32} \mathrm{P}$-labelled substrates. The fluxes determined with ${ }^{14} \mathrm{C}$ - and ${ }^{32} \mathrm{P}$-labelled substrates are also compared at chemical equilibrium. 4 . With rabbit muscle phosphoglucomutase the results of these tests were completely consistent with mechanism 1 and unequivocally excluded any substantial part of the reaction proceeding by mechanism 2 or mechanism 3. Evidence was also obtained for an isomerization of the phosphoenzyme with an apparent rate constant about $4.5 \times 10^{7} \mathrm{sec} .^{-1}$. Taking into account the activity coefficients of the substrates the true rate constant appears to be about one-sixth of this value. 5. Isotope effects and non-ideal behaviour of the solutions are discussed and the activity coefficients of the substrates are shown to be equal by measurement of the depression of freezing point. It is concluded that these factors do not influence the tests significantly. 6. Alternative mechanisms are considered and it is concluded that the tests show that the glucose residue is transferred directly, that the phosphate is transferred indirectly with one intermediate phosphate, and that there is an isomerization of the free phosphoenzyme without reference to any other details of the reaction. Further, no assumptions are required about the constancy of rate constants. 7. The relative merits of induced transport and product inhibition for detecting isomerization of the enzyme are discussed. It is concluded that the induced-transport test is more sensitive and that its interpretation is less equivocal. 8. The application of the tests to other enzyme systems is briefly considered.
\end{abstract}

Phosphoglucomutase (EC 2.7.5.1) from rabbit muscle is believed to catalyse the conversion of G1P* into G6P by the mechanism (mechanism 1) shown in Scheme 1 (Ray \& Roscelli, 1964a,b, $1966 a, b$; Ray, Roscelli \& Kirkpatrick, 1966 ; Najjar \& Pullman, 1954). When a molecule of G1P is converted into G6P by this mechanism the phosphate from the GIP is transferred to the enzyme and the phosphate attached to the enzyme is transferred to the 6-position of the substrate. There is thus an intermediate phosphate involved in phosphate transfer. This mechanism was proposed because of the requirement of the enzyme

*Abbreviations: G1P, glucose 1-phosphate; G6P, glucose 6-phosphate; G1P6P, glucose 1,6-diphosphate. for G1P6P as a cofactor, because of the existence of a phosphorylated form of the enzyme and because the reciprocal of the initial reaction velocity plotted against the reciprocal of the G1P concentration, at different G1P6P concentrations, gave a series of parallel lines. A series of parallel lines was also obtained when the reciprocal of the initial reaction velocity was plotted against the reciprocal of the G1P6P concentration at different G1P concentrations. Free G1P6P in solution, as is indicated in Scheme 1, did not appear to be an obligatory intermediate in the reaction since 32P-labelled enzyme incubated for a short period with a large excess of G1P and G1P6P transferred most of its label to the monophosphates and

Bioch. 1968, 110 


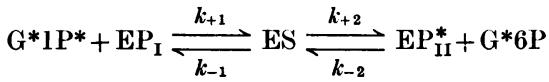

$$
\begin{aligned}
& \mathrm{EP}_{\mathrm{II}}^{*} \underset{k_{-3}}{\stackrel{k_{+3}}{\rightleftharpoons}} \mathrm{EP}_{\mathrm{I}}^{*} \\
& \mathrm{G} 1 \mathrm{P}+\mathrm{EP}_{\mathrm{I}}^{*} \underset{k_{-1}}{\stackrel{k_{+1}}{\rightleftharpoons}} \mathrm{ES} \underset{k_{-2}}{\stackrel{k_{+2}}{\rightleftharpoons}} \mathrm{EP}_{1 \mathrm{I}}+\mathrm{G}_{6} \mathrm{P}^{*} \\
& \mathrm{EP}_{\mathrm{II}} \stackrel{\stackrel{k_{+3}}{\rightleftharpoons}}{\underset{k_{-3}}{\rightleftharpoons}} \mathrm{EP}_{\mathrm{I}}
\end{aligned}
$$

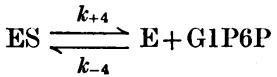

Scheme 1. Scheme (mechanism 1) to show transfer of labelled glucose and labelled phosphate from G1P to G6P by a mechanism involving the transfer of the phosphate from the substrate to the phosphoenzyme. ES represents the enzyme-substrate complex; $\mathbf{E P}_{\mathbf{I}}$ and $\mathbf{E P}_{\mathrm{II}}$ represent different forms of the phosphoenzyme, and $\mathrm{E}$ represents the dephosphoenzyme. $k_{+1}, k_{+2}$ etc. are rate constants. $\mathrm{G}^{*}$ and $\mathrm{P}^{*}$ represent labelled molecules. The labelled glucose is transferred directly whereas two G1P molecules have to react in succession with the enzyme to transfer the phosphate.

particularly to G6P (Ray \& Roscelli, 1964a). Two forms of the phosphorylated enzyme, designated $\mathrm{EP}_{\mathrm{I}}$ and $\mathrm{EP}_{\mathrm{II}}$, are shown since the mechanism would seem to demand their existence. However, Ray \& Roscelli (1964b) were unable to find any evidence for phosphoenzyme isomerization from product inhibition and they concluded that the apparent rate constant for the interconversion of the species, if they should exist, must be in excess of $10^{7} \mathrm{sec}^{-1}$. The corresponding limit for the true rate constant will be somewhat lower than this since these authors did not take into account the non-ideal behaviour of the solutions.

Although the transfer of ${ }^{32} \mathrm{P}$-labelled phosphate from the phosphoenzyme to the substrate strongly suggests that the phosphate attached to the enzyme takes part in the reaction in the way shown in Scheme 1 (mechanism 1), reaction times of the order of seconds were used and it is conceivable that over these relatively long periods the phosphate may have been transferred from the enzyme by side reactions. Thus in the normal enzyme reaction there could be a direct transfer of phosphate from the 1-position to the 6-position with the phosphate attached to the enzyme performing some indirect role such as the formation of a pyrophosphate bond. This mechanism is referred to below as mechanism 2. Such a direct transfer of phosphate might explain why the enzyme still shows some activity in the absence of G1P6P (Ray \& Roscelli, 1964a).

Unlike the enzyme from rabbit muscle, the phosphoglucomutases obtained by Hanabusa,

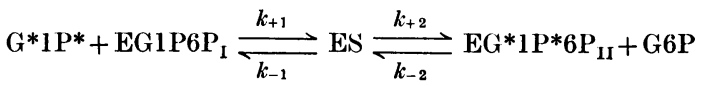

$$
\begin{aligned}
& \mathrm{EG}^{*} 1 \mathrm{P}^{*} 6 \mathrm{P}_{\mathrm{II}} \underset{k_{-3}}{\stackrel{k_{+3}}{\rightleftharpoons}} \mathrm{EG} 1 \mathrm{P}^{*} 6 \mathrm{P}_{\mathrm{I}} \\
& \mathrm{G} 1 \mathrm{P}+\mathrm{EG}^{*} 1 \mathrm{P}^{*} 6 \mathrm{P}_{\mathrm{I}} \underset{k_{-1}}{\stackrel{k_{+1}}{\rightleftharpoons}} \mathrm{ES} \underset{k_{-2}}{\stackrel{k_{+2}}{\rightleftharpoons}} \mathrm{EG} 1 \mathrm{P} 6 \mathrm{P}_{\mathrm{II}}^{*}+\mathrm{G} 6 \mathrm{P} \\
& \text { EG1P6P }{ }_{\mathrm{II}}^{*} \underset{k_{-3}}{\stackrel{k_{+3}}{\rightleftharpoons}} \text { EG1P6P I }
\end{aligned}
$$

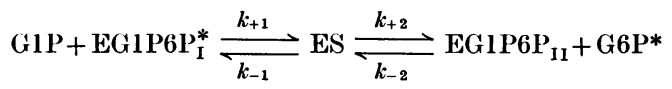

$$
\begin{aligned}
& \text { EGlP6P } 11 \underset{k_{-3}}{\stackrel{k_{+3}}{\rightleftharpoons}} \text { EG1P6P }
\end{aligned}
$$

Scheme 2. Scheme (mechanism 3) to show transfer of labelled glucose and labelled phosphate from G1P to G6P by a mechanism involving the intermolecular transfer of phosphate from G1P6P to GIP. EG1P6P 1 and EG1P6P represent different forms of the enzyme-G1P6P complex. $\mathrm{G}^{*}$ and $\mathrm{P} *$ represent labelled molecules. $k_{+1}, k_{+2}$ etc. are rate constants. The reaction is supposed to take place in the presence of excess of G1P6P, so that dissociation of the enzyme-G1P6P complex can be ignored. Two molecules of GIP have to react in succession with the enzyme to transfer the labelled glucose. At least three molecules of GlP have to react to transfer the phosphate.

Dougherty, del Río, Hashimoto \& Handler (1966) from Micrococcus lysodeikticus and Bacillus cereus could not be isolated in phosphorylated forms although G1P6P was still required as a cofactor. These authors also found that the kinetics of these enzymes differed from that of rabbit muscle, in that the reciprocal of the initial velocities plotted against the reciprocal of the G1P concentration at different G1P6P concentrations gave a series of intersecting lines, indicating that the apparent $K_{m}$ for G1P was independent of G1P6P concentration; and a similar series of intersecting lines was obtained when the reciprocal of the initial velocity was plotted against the reciprocal of the G1P6P concentration at different G1P concentrations. They therefore proposed that these enzymes catalyse an intermolecular transfer of phosphate from G1P6P to the substrates as is shown in Scheme 2 (mechanism 3). In this mechanism there is not only an indirect transfer of phosphate, which involves two intermediate phosphate groups, but an indirect transfer of the glucose with a single intermediate glucose molecule.

It is surprising that the mechanisms for phosphoglucomutases from different sources should be so different. Mechanism 1 appears not to be a possible mechanism for the phosphoglucomutases from $M$. lysodeikticus and B. cereus because these enzymes do not exist in phosphorylated forms, and because 
the $K_{m}$ for G1P is unaffected by G1P6P concentration. However, if the phosphoenzyme spontaneously dephosphorylated with the same rate constant as the dissociation of G1P6P from the enzymesubstrate complex then appropriate kinetics would be obtained and the absence of a phosphorylated form of the enzyme would also be explained. Alternatively, one of the phosphates of the enzymeG1P6P complex shown in Scheme 2 (mechanism 3) might play the same role as the phosphate in the phosphoenzyme. The direct transfer of phosphate would clearly be a possible mechanism for all types of phosphoglucomutases. Mechanism 3 has been rejected as a possible mechanism for rabbit muscle phosphoglucomutase because parallel lines were obtained when the reciprocals of the initial velocities were plotted against the reciprocals of the G1P concentration at different G1P6P concentrations. However, if the free enzyme had a much greater affinity for G1P6P than had the enzyme combined with substrate then it can be shown that the kinetics of the rabbit muscle enzyme may be obtained. If mechanism 3 describes the mechanism of rabbit muscle phosphoglucomutase, the phosphoenzyme must represent an artifact of the separation procedure, since the effective agent will be the enzyme-G1P6P complex, and it may be noted that Hanabusa et al. (1966) have reported the isolation of the enzyme with a rather tightly bound molecule of G1P6P.

It may also be relevant that Brody \& Tatum (1967) found that phosphoglucomutase from the wild forms of Neurospora crassa gave kinetics resembling those of the $M$. lysodeikticus and $B$. cereus enzymes, whereas the enzyme derived from a mutated strain gave kinetics resembling those of the rabbit enzyme. Unless two enzymes are involved it would seem unlikely that a mutation would alter the reaction mechanism.

Thus all three mechanisms would seem to be possible for phosphoglucomutase. The flux approach of Britton (1965a, 1966a,b, 1967a,b) offers a method of distinguishing between these various possibilities and also provides a very sensitive method of detecting any isomerization of the free enzyme. It is used in the present paper to show that in rabbit muscle phosphoglucomutase the glucose moiety is directly transferred between the substrates and that phosphate is transferred indirectly with one intermediate phosphate. Mechanism 1 thus represents the only possible mechanism. Evidence is also obtained for the existence of two forms of the free phosphorylated enzyme. The apparent rate constant for the interconversion of the species is about $4.5 \times 10^{7} \mathrm{sec}^{-1}$, but when the activity coefficients of the substrates are taken into account the true rate constant would appear to be about one-sixth of this value.

The role of $\mathrm{Mg}^{2+}$, an essential cofactor, has not been considered in the above discussion. Ray \& Roscelli (1966a,b) and Ray et al. (1966) have extensively investigated this aspect of the reaction and have obtained very strong evidence that the $\mathrm{Mg}^{2+}$ remains attached to the enzyme during the whole of the enzymic process. All the experimental work described below was carried out in the presence of excess of $\mathrm{Mg}^{2+}$.

\section{THEORY}

In the following, the term flux is used in the way defined by Britton (1966b). The flux of phosphate from G1P to G6P is the actual number of phosphate groups transferred from the 1-position to the 6-position of the glucose in unit time under the particular conditions of concentrations, temperature etc. that are employed, and the flux of glucose from G1P to G6P is the rate of transfer of glucose from G1P to G6P.

Derivation of flux equations. (a) Flux equations for mechanism 1. In a previous publication (Britton, 1966b) the fluxes for a reaction of the type:

$$
\mathbf{R X}+\mathbf{R}^{\prime} \rightleftharpoons \mathbf{R}+\mathbf{R}^{\prime} \mathbf{X}
$$

were calculated for a mechanism in which the enzyme forms a stable intermediate with the group transferred. Thus the initial reaction is the transference of $\mathrm{X}$ from $\mathrm{RX}$ to the enzyme to form an EX complex. The EX complex then isomerizes to a form that can transfer $X$ to $R^{\prime}$ to yield $R^{\prime} X$. Finally the free enzyme isomerizes to its original form. In the previous publication (Britton, 1966b) $\mathrm{RX}=\mathrm{A}$, $R^{\prime}=B, R=C$ and $R^{\prime} X=D$. Considering mechanism 1 (Scheme 1), and if $A=B=G 1 P$ and $C=D=G 6 P$, then at any one instant in time one-half of the EP molecules may be regarded as transferring phosphate from $A$ to $D$ and the other half as transferring phosphate from B to C. Thus by comparison with eqns. 24-29 in Britton (1966b): flux of phosphate from GIP to G6P $=2 \times$ flux of $A$ to $D$; flux of phosphate from G6P to G1P $=2 \times$ flux of D to A; flux of glucose residues from $\mathrm{GlP}$ to $\mathrm{G} 6 \mathrm{P}=2 \times$ flux of $A$ to $\mathrm{C}$; flux of glucose residues from G6P to $\mathrm{GlP}=2 \times$ flux of $\mathrm{C}$ to $\mathrm{A}$; $\left(E P_{I}\right)=2 E_{A}=2 E_{B}$ and $\left(E P_{I I}\right)=2 E_{C}=2 E_{D}$, where $\left(E P_{I}\right)$, $\left(\mathbf{E P}_{\mathrm{II}}\right), \mathbf{E}_{\mathrm{A}}, \mathbf{E}_{\mathrm{B}}, \mathbf{E}_{\mathrm{C}}$ and $\mathbf{E}_{\mathrm{D}}$ represent the quantities of the enzyme species; $[\mathrm{G} 1 \mathrm{P}]=\mathrm{A}=\mathrm{B}$ and $[\mathrm{G} 6 \mathrm{P}]=\mathrm{C}=\mathrm{D}$, where the symbols represent concentrations; and $k_{+1}=a=g$, $k_{-1}=b=h, k_{+2}=c=k, k_{-2}=d=l, k_{+3}=e=m$ and $k_{-3}=f=n$.

Substituting:

Flux of phosphate from G1P to G6P $=\frac{k_{+1}{ }^{2} k_{+2}{ }^{2} k_{+3}[\mathrm{G} 1 \mathrm{P}]^{2}\left(\mathrm{EP}_{\mathrm{I}}\right)}{k_{+1} k_{+2} k_{+3}\left(k_{-1}+k_{+2}\right)[\mathrm{GlP}]+k_{-1} k_{-2} k_{-3}\left(k_{-1}+k_{+2}\right)[\mathrm{G} 6 \mathrm{P}]+k_{+1} k_{+2} k_{-1} k_{-2}[\mathrm{G} 1 \mathrm{P}][\mathrm{G} 6 \mathrm{P}]}$ 
Flux of phosphate from G6P to GIP $=\frac{k_{-1} 2 k_{-2}{ }^{2} k_{-3}[\mathrm{G} 6 \mathrm{P}]^{2}\left(\mathrm{EP}_{\mathrm{II}}\right)}{k_{+1} k_{+2} k_{+3}\left(k_{-1}+k_{+2}\right)[\mathrm{G} 1 \mathrm{P}]+k_{-1} k_{-2} k_{-3}\left(k_{-1}+k_{+2}\right)[\mathrm{G} 6 \mathrm{P}]+k_{+1} k_{+2} k_{-1} k_{-2}[\mathrm{GlP}][\mathrm{G} 6 \mathrm{P}]}$

Flux of glucose residues from G1P to G6P

$$
=\frac{k_{+1} k_{+2}[\mathrm{GlP}]\left(\mathrm{EP}_{\mathrm{I}}\right)}{k_{-1}+k_{+2}}
$$

and

Flux of glucose residues from G6P to GlP

$$
=\frac{k_{-1} k_{-2}[\mathrm{G} 6 \mathrm{P}][\mathrm{EP} \text { II }]}{k_{-1}+k_{+2}}
$$

Further, in the steady state:

$$
\left(\mathrm{EP}_{\mathrm{I}}\right)=\frac{(\mathrm{E})\left\{k_{+3}\left(k_{-1}+k_{+2}\right)+k_{-1}\right.}{D_{1}} \underline{\left.k_{-2}[\mathrm{G} 6 \mathrm{P}]\right\}}
$$

and

$$
\left(\mathrm{EP}_{\mathrm{II}}\right)=\frac{(\mathrm{E})\left\{k_{-3}\left(k_{-1}+k_{+2}\right)+k_{+1} k_{+2}[\mathrm{G} 1 \mathrm{P}]\right\}}{D_{1}}
$$

where:

$$
\begin{aligned}
D_{1}= & k_{+1}\left(k_{+2}+k_{+3}\right)[\mathrm{G} 1 \mathrm{P}]+k_{-2}\left(k_{-1}+k_{-3}\right)[\mathrm{G} 6 \mathrm{P}] \\
& +k_{+1} k_{-2}[\mathrm{G} 1 \mathrm{P}][\mathrm{G} 6 \mathrm{P}]+\left(k_{+3}+k_{-3}\right)\left(k_{-1}+k_{+2}\right) \\
& +\frac{k_{+4}}{k_{-4}[\mathrm{GlP} 6 \mathrm{P}]}\left(k_{+3}[\mathrm{G} 1 \mathrm{P}]+k_{-3}[\mathrm{G} 6 \mathrm{P}]\right. \\
& \left.+k_{+1} k_{-2}[\mathrm{G} 1 \mathrm{P}][\mathrm{G} 6 \mathrm{P}]\right)
\end{aligned}
$$

and $(E)$ is the total quantity of enzyme.

The rate constants must also be related by the expression:

$$
K k_{+1} k_{+2} k_{+3}=k_{-1} k_{-2} k_{-3}
$$

where $K$ is the equilibrium constant.

From eqns. (1), (2), (5), (6) and (7):

Flux of phosphate from GIP to G6P

Flux of phosphate from G6P to GIP

$$
\begin{aligned}
& =\frac{[\mathrm{GlP}]^{2}\left(\mathrm{EP}_{\mathrm{I}}\right) k_{-3}}{[\mathrm{G} 6 \mathrm{P}]^{2} K^{2}\left(\mathrm{EP}_{\mathrm{II}}\right) k_{+3}} \\
& =\frac{[\mathrm{GlP}]^{2}(1+\alpha K[\mathrm{G} 6 \mathrm{P}])}{[\mathrm{G} 6 \mathrm{P}]^{2} K^{2}(1+\alpha[\mathrm{GlP}])}
\end{aligned}
$$

From eqns. (3), (4), (5), (6) and (7):

Flux of glucose residues from G1P to G6P Flux of glucose residues from G6P to G1P

$$
\begin{aligned}
& =\frac{[\mathrm{GlP}]\left(\mathrm{EP}_{\mathrm{I}}\right) k_{-3}}{[\mathrm{G} 6 \mathrm{P}] K\left(\mathrm{EP}_{\mathrm{II}}\right) k_{+3}} \\
& =\frac{[\mathrm{GlP}](1+\alpha K[\mathrm{G} 6 \mathrm{P}])}{[\mathrm{G} 6 \mathrm{P}] K(1+\alpha[\mathrm{GlP}])}
\end{aligned}
$$

And at chemical equilibrium from eqns. (1), (3) and (7):

$\frac{\text { Flux of glucose residues from G1P to G6P }}{\text { Flux of phosphate from G1P to G6P }}=2+\alpha[$ G1P $]$

where:

$$
\alpha=k_{+1} k_{+2} / k_{-3}\left(k_{-1}+k_{+2}\right)
$$

Since $\alpha$ is inversely related to $k_{-3}, \alpha$ will be zero when the isomerization of the phosphoenzyme is not rate-limiting.

(b) Flux equations when there is direct transfer of phosphate (mechanism 2). For a mechanism similar to mechanism 1 but in which there is a direct transfer of phosphate between the 1-position and the 6-position (i.e. mechanism 2) the expressions for both the glucose and phosphate fluxes will be those given for the glucose fluxes in Scheme 1. The ratio of the glucose flux to the phosphate flux will be unity. Similar flux-ratio equations would be obtained if the effective agent were an enzymeG1P6P complex as in mechanism 3 (Scheme 2).

(c) Flux equations for mechanism 3. For mechanism 3 an entirely analogous method of calculation as for mechanism 1 may be used and it is possible to write:

Flux of phosphate from G1P to G6P

$$
=\frac{k_{+1}{ }^{3} k_{+2}{ }^{3} k_{+3}{ }^{2}[\mathrm{G} 1 \mathrm{P}]^{3}\left(\mathrm{EG} 1 \mathrm{P} 6 \mathrm{P}_{\mathrm{I}}\right)}{D_{2}}
$$

Flux of phosphate from G6P to GIP

$$
=\frac{k_{-1} k_{-2}{ }^{3} k_{-3}{ }^{2}[\mathrm{G} 6 \mathrm{P}]^{3}\left(\mathrm{EG} 1 \mathrm{P} 6 \mathrm{P}_{\mathrm{II}}\right)}{D_{2}}
$$

where:

$$
\begin{gathered}
D_{2}=k_{+1}{ }^{2} k_{+2}{ }^{2} k_{+3}{ }^{2}\left(k_{-1}+k_{+2}\right)[\mathrm{G} 1 \mathrm{P}]^{2}+k_{-1}{ }^{2} k_{-2} 2 k_{-3}{ }^{2}\left(k_{-1}+k_{+2}\right)[\mathrm{G} 6 \mathrm{P}]^{2}+k_{+1} k_{+2} k_{+3} k_{-1} k_{-2} k_{-3}\left(k_{-1}+k_{+2}\right)[\mathrm{G} 1 \mathrm{P}][\mathrm{G} 6 \mathrm{P}] \\
+k_{+1} k_{+2} k_{-1} k_{-2} k_{-3}[\mathrm{GlP}][\mathrm{G} 6 \mathrm{P}]^{2}+k_{+1} k_{+2} k_{+3} k_{-1} k_{-2}[\mathrm{GlP}]^{2}[\mathrm{G} 6 \mathrm{P}]
\end{gathered}
$$

Flux of glucose from G1P to G6P $=\frac{k_{+1}{ }^{2} k_{+2}{ }^{2} k_{+3}[\mathrm{G} 1 \mathrm{P}]^{2}\left(\mathrm{EG} 1 \mathrm{P} 6 \mathrm{P}_{\mathrm{I}}\right)}{k_{+1} k_{+2} k_{+3}\left(k_{-1}+k_{+2}\right)[\mathrm{GIP}]+k_{-1} k_{-2} k_{-3}\left(k_{-1}+k_{+2}\right)[\mathrm{G} 6 \mathrm{P}]+k_{+1} k_{+2} k_{-1} k_{-2}[\mathrm{GlP}][\mathrm{G} 6 \mathrm{P}]}$

and

Flux of glucose from G6P to G1P $=\frac{k_{-1}{ }^{2} k_{-2}{ }^{2} k_{-3}[\mathrm{G} 6 \mathrm{P}]^{2}\left(\mathrm{EG} 1 \mathrm{P} 6 \mathrm{P}_{\mathrm{II}}\right)}{k_{+1} k_{+2} k_{+3}\left(k_{-1}+k_{+2}\right)[\mathrm{GIP}]+k_{-1} k_{-2} k_{-3}\left(k_{-1}+k_{+2}\right)[\mathrm{G} 6 \mathrm{P}]+k_{+1} k_{+2} k_{-1} k_{-2}[\mathrm{GIP}][\mathrm{G} 6 \mathrm{P}]}$ 
In addition in the presence of excess of G1P6P:

$$
\left(\mathrm{EG} 1 \mathrm{P} 6 \mathrm{P}_{\mathrm{I}}\right)=\frac{(\mathrm{E})\left\{k_{+3}\left(k_{-1}+k_{+2}\right)+k_{-1} k_{-2}[\mathrm{G} 6 \mathrm{P}]\right\}}{k_{+1}\left(k_{+2}+k_{+3}\right)[\mathrm{GIP}]+k_{-2}\left(k_{-1}+k_{-3}\right)[\mathrm{G} 6 \mathrm{P}]+k_{+1} k_{-2}[\mathrm{GlP}][\mathrm{G} 6 \mathrm{P}]+\left(k_{+3}+k_{-3}\right)\left(k_{-1}+k_{+2}\right)}
$$

and

$$
\left(\mathrm{EG1P} 6 \mathrm{P}_{\mathrm{II}}\right)=\frac{(\mathrm{E})\left\{k_{-3}\left(k_{-1}+k_{+2}\right)+k_{+1} k_{+2}[\mathrm{GIP}]\right\}}{k_{+1}\left(k_{+2}+k_{+3}\right)[\mathrm{GIP}]+k_{-2}\left(k_{-1}+k_{-3}\right)[\mathrm{G} 6 \mathrm{P}]+k_{+1} k_{-2}[\mathrm{GIP}][\mathrm{G} 6 \mathrm{P}]+\left(k_{+3}+k_{-3}\right)\left(k_{-1}+k_{+2}\right)}
$$

where (E) is the total quantity of enzyme.

The rate constants must also be related as in eqn. (7).

From eqns. (12), (13), (16), (17) and (7):

Flux of phosphate from G1P to G6P

Flux of phosphate from G6P to G1P

$$
\begin{aligned}
& =\frac{[\mathrm{GlP}]^{3}\left(\mathrm{EG} 1 \mathrm{P} 6 \mathrm{P}_{\mathrm{I}}\right) k_{-3}}{[\mathrm{G} 6 \mathrm{P}]^{3} K^{3}\left(\mathrm{EG} 1 \mathrm{P} 6 \mathrm{P}_{\mathrm{II}}\right) k_{+3}} \\
& =\frac{[\mathrm{GlP}]^{3}(1+\alpha K[\mathrm{G} 6 \mathrm{P}])}{[\mathrm{G} 6 \mathrm{P}]^{3} K^{3}(1+\alpha[\mathrm{GlP}])}
\end{aligned}
$$

From eqns. (14), (15), (16), (17) and (7):

Flux of glucose from G1P to G6P

Flux of glucose from G6P to GIP

$$
\begin{aligned}
& =\frac{[\mathrm{GlP}]^{2}\left(\mathrm{EG} 1 \mathrm{P} 6 \mathrm{P}_{\mathrm{I}}\right) k_{-3}}{[\mathrm{G} 6 \mathrm{P}]^{2} K^{2}\left(\mathrm{EG} 1 \mathrm{P} 6 \mathrm{P}_{\mathrm{II}}\right) k_{+3}} \\
& =\frac{[\mathrm{GlP}]^{2}(1+\alpha K[\mathrm{G} 6 \mathrm{P}])}{[\mathrm{G} 6 \mathrm{P}]^{2} K^{2}(1+\alpha[\mathrm{G} 1 \mathrm{P}])}
\end{aligned}
$$

And at chemical equilibrium from eqns. (12), (14) and (7):

$\frac{\text { Flux of glucose from G1P to G6P }}{\text { Flux of phosphate from G1P to G6P }}=\frac{3+2 \alpha[\mathrm{GlP}]}{2+\alpha[\mathrm{GlP}]}$

where $\alpha$ is defined as in eqn. (11) but with the rate constants referring to mechanism 3 (Scheme 2).

At high concentrations of substrates or in solutions of high electrolyte strength the activity coefficients of G1P and G6P should be introduced into the flux equations. The activity coefficients of the different enzymic species should also be included in relation to the appropriate rate constants, but if the activity coefficients of all the enzymic species are the same these coefficients cancel. The latter assumption may not necessarily be true if the electrical charge on the enzyme changes on the addition of substrate, and as a consequence the apparent rate constants may change in solutions of high electrolyte strength. However, except for the numerical value of $\alpha$, the flux-ratio equations will be unaltered.

The flux-ratio equations for mechanisms 1 and 2 are independent of the G1P6P concentration. For mechanism 3 the flux-ratio equations will remain of the same form at low G1P6P concentrations, but the significance of the rate constants may change somewhat if an appreciable part of the reaction takes place by the substrates combining with the free enzyme and with the G1P6P subsequently adding to the enzyme-substrate complex. The latter is unlikely to be the case if G1P6P dissociates only occasionally from the enzyme except at very low substrate concentrations.

In deriving the flux equations it has been assumed either that the free G1P6P in solution exchanges rapidly and comes into a steady state or that the rate of exchange of G1P6P is negligible. The experimental result lies between the two extremes, but as the quantity of G1P6P present is small the effect is relatively unimportant. It is discussed in the Results section.

When the isomerization of the enzyme is not ratelimiting an alternative way of deriving the flux-ratio equations is by the probability method of Hodgkin \& Keynes (1955).

Significance of the fux ratios. If GIP molecules are converted into G6P molecules independently then the flux ratio should obey the Independence relationship (Britton, $1965 a)$.

Thus:

$$
\frac{\text { Flux of G1P to } \mathrm{G} 6 \mathrm{P}}{\text { Flux of } \mathrm{G} 6 \mathrm{P} \text { to } \mathrm{G} 1 \mathrm{P}}=\frac{f_{\mathrm{G} 1 \mathrm{P}}[\mathrm{G} 1 \mathrm{P}]}{f_{\mathrm{G} 6 \mathrm{P}}[\mathrm{G} 6 \mathrm{P}] K}
$$

where $f_{\mathrm{G} 1 \mathrm{P}}$ and $f_{\mathrm{G} 6 \mathrm{P}}$ represent the activity coefficients of G1P and G6P respectively and the other symbols are as defined above.

Consider a net conversion of G1P into G6P. For mechanism 1 the glucose flux ratio (eqn. 9) should be less than that expected from the Independence relationship, and the chemical equivalent of exchange diffusion should occur unless $\alpha[\mathrm{G} 1 \mathrm{P}]$ and $\alpha K[\mathrm{G} 6 \mathrm{P}]$ are very small, when the Independence relationship should be obeyed. The same will be true for the phosphate flux ratio if there is a direct transfer of phosphate as in mechanism 2. However, for mechanism 1 the phosphate flux ratio (eqn. 8) should exceed that to be expected from the Independence relationship, so that the chemical analogue of the long-pore effect should be found. For mechanism 3 both the glucose flux ratio (eqn. 19) and the phosphate flux ratio (eqn. 18) should show the long-pore effect. Examination of the reaction for the chemical analogues of exchange diffusion and the long-pore effect should thus provide a method of distinguishing between the models. The ratio of the glucose flux to the phosphate flux at chemical equilibrium will provide a further method of making a distinction, since the ratio will be $1: 1$ if there is a direct transfer of phosphate (mechanism 2), but will be greater than 1:1 for mechanisms 1 and 3 (eqns. 10 and 20). In contrast, the expressions for the net fluxes for all three models are identical in the presence of excess of G1P6P. At lower concentrations of G1P6P the expressions for the net fluxes for mechanisms 1 and 2 remain identical but for mechanism 3 the effect of a low G1P6P concentration will depend on the relative values of the rate constants, as has been discussed in the introduction.

To examine an $\mathrm{A} \rightleftharpoons \mathrm{B}$ reaction of the present type for the chemical analogue of exchange diffusion or the long-pore effect, it is convenient to use tests based on induced transport (Britton, 1963, 1965a, 1966 $b$; and see the Appendix). To carry out these tests appropriately labelled substrate (G1P or G6P) is allowed to come into equilibrium in the presence of the enzyme and then unlabelled substrate (GIP) is added. If activity coefficients and isotope effects are ignored, then, if the flux ratio obeys the Independence 
relationship, the distribution of radioactivity between the two substrates will remain unchanged during the reaction. If the reaction shows the chemical analogue of exchange diffusion there will be a transient flow of labelled material in a direction opposite to the net flow; by analogy with the permeability studies this may be called counter-transport. Finally, if the reaction shows the chemical analogue of the long-pore effect there will be a transient flow of labelled material in the same direction as the net flow; this may be termed co-transport. The influence of isotope effects and non-ideal behaviour of solutions on these tests is considered in the Discussion section.

\section{MATERIALS AND METHODS}

Reagents and materials. G1P, G6P and G1P6P, the monophosphates as the sodium salts and the diphosphate as the cyclohexylammonium salt, were obtained from C. F. Boehringer und Soehne G.m.b.H. (Mannheim, Germany). Rabbit skeletal-muscle phosphoglucomutase was purchased from the same source as a crystalline suspension in $0.25 \mathrm{M}-\left(\mathrm{NH}_{4}\right)_{2} \mathrm{SO}_{4}$. The enzyme was separated from $\left(\mathrm{NH}_{4}\right)_{2} \mathrm{SO}_{4}$ by placing it on a short column of Sephadex G-25 that had been equilibrated with $9.6 \mathrm{~mm}$-tris-chloride buffer, $\mathrm{pH} 7 \cdot 4$, and by eluting with the same buffer. The enzyme was used without further purification, but was activated immediately before use by adding a histidine-tris buffer, $\mathrm{pH7 \cdot 4}$, to give final concentrations of histidine $(40 \mathrm{mM})$, tris $(12 \mathrm{mM}), \mathrm{MgCl}_{2}(1 \cdot 1 \mathrm{mM})$ and gelatin $(50 \mu \mathrm{g} . / \mathrm{ml}$.) and incubating for $10 \mathrm{~min}$. at $30^{\circ}$ as described by Ray \& Roscelli $(1964 a, 1966 a)$. When assayed according to the method of Ray \& Roscelli (1964a) an activity of about 50-100 units/ $\mu \mathrm{g}$. was obtained, which may be compared with the value of $105 \mathrm{units} / \mu \mathrm{g}$. obtained by Ray \& Roscelli (1964a) for their most active preparations.

Solutions of G1P and G6P were adjusted to $\mathrm{pH} \mathrm{7 \cdot 4}$ with $\mathrm{HCl}$ at a glass electrode.

[G-14C]G6P was obtained from The Radiochemical Centre (Amersham, Bucks.) and was used without further purification.

[32P]G1P was prepared by the following procedure. A 1 mc sample of sodium [ $\left.{ }^{32} \mathrm{P}\right]$ phosphate, supplied as a sterilized solution, pH3, by The Radiochemical Centre, was evaporated to dryness in a vacuum desiccator over $\mathrm{NaOH}$. Then $0.2 \mathrm{ml}$. of $10 \mathrm{~mm}$-sodium phosphate buffer, $\mathrm{pH} 7$, was added, followed by $0.1 \mathrm{ml}$. of aq. $4 \%(\mathrm{w} / \mathrm{v})$ glycogen solution and $0.1 \mathrm{ml}$. of rabbit muscle phosphorylase solution [supplied by Sigma Chemical Co. (St Louis, Mo., U.S.A.) as a $2 \mathrm{mg}$. $/ \mathrm{ml}$. suspension in $1.5 \mathrm{~mm}$-EDTA, $1.92 \mathrm{~mm}$ sodium glycerophosphate adjusted to $\mathrm{pH} 6.6$ with acetic acid and $0 \cdot 1 \mathrm{M}-\mathrm{NaF}$; the activity was $1434 \mathrm{units} / \mathrm{mg}$. (Cori, Cori \& Green, 1943)]. After incubation at $30^{\circ}$ for $90 \mathrm{~min}$. the mixture was evaporated to dryness in a vacuum desiccator. It was then dissolved in a small quantity of water and chromatographed successively once with butan1-ol-acetic acid-water (12:3:5, by vol.) (as a 2in.-wide strip) and twice with 2-methoxyethanol-othyl methyl ketone-aq. $3 \mathrm{~N}-\mathrm{NH}_{3}$ (7:2:3, by vol.) (Mortimer, 1952) on Whatman no. 4 paper.

All glassware was cleaned with Decon 75 (A. Gallenkamp and Co. Ltd., London, E.C. 2) before use.

Analytical methods. Total enzyme protein was determined from the extinction at $278 \mathrm{~m} \mu$ by using $E_{1}^{1 \%} \mathrm{~cm} .7 \cdot 70$ (Najjar, 1948).
GlP was determined by a slight modification of Bartlett's (1959) phosphate method as described by Ray \& Roscelli $(1964 a)$. Samples $(0 \cdot 1 \mathrm{ml}$.) were taken from the reaction mixture and added to $4 \mathrm{ml}$. of $0 \cdot 18 \%(\mathrm{w} / \mathrm{v})$ ammonium molybdate in $\mathrm{N}-\mathrm{H}_{2} \mathrm{SO}_{4}$ in stoppered test tubes. Then $0 \cdot 2 \mathrm{ml}$. of reducing solution $(2 \cdot 4 \mathrm{~g}$. of anhydrous sodium sulphite, $12 \mathrm{~g}$. of sodium metabisulphite and $0 \cdot 2 \mathrm{~g}$. of 1-amino-2naphthol-4-sulphonic acid in $100 \mathrm{ml}$. of water) was added and the test tubes were immersed in a boiling-water bath for $10 \mathrm{~min}$. The extinctions were measured at $830 \mathrm{~m} \mu$. Hydrolysis of the G1P and colour development take place at the same time in this procedure.

[32P]G1P was determined by a variation of the method of Ray et al. (1966) based on the phosphate precipitation technique of Sugino \& Miyoshi (1964). A mixture of $57 \mathrm{~mm}$-ammonium molybdate, $74 \mathrm{~mm}$-triethylamine and $0.9 \mathrm{~N}-\mathrm{HClO}_{4}(2: 1: 1$, by vol.) was prepared shortly before use and $0.4 \mathrm{ml}$. portions were placed in $15 \mathrm{ml}$. conical centrifuge tubes. Samples $(0 \cdot 1 \mathrm{ml}$.) taken from the reaction mixture were added to the centrifuge tubes followed by $0.5 \mathrm{ml}$. of a solution containing G1P $(0.4 \mathrm{~mm})$ and G6P $(0.4 \mathrm{~mm})$. The centrifuge tubes were placed in a boilingwater bath for $10 \mathrm{~min}$., cooled thoroughly in cold running water and kept for at least $1 \mathrm{hr}$. at $4^{\circ}$. After centrifuging at $2000 \mathrm{~g}$ for $20 \mathrm{~min}$., the supernatants were removed and the precipitates washed three times, without disturbing the compacted material, with a mixture consisting of $57 \mathrm{~mm}$ ammonium molybdate, $74 \mathrm{~mm}$-triethylamine, $0.9 \mathrm{~N}-\mathrm{HClO}_{4}$ and water $(2: 1: 1: 6$, by vol.) to which a trace of phosphate had been added. The precipitates were then dissolved with gentle warming in $0.2 \mathrm{ml}$. of $\mathrm{N}-\mathrm{NaOH}$ and each solution was transferred quantitatively with three washings of $0.2 \mathrm{ml}$. of water to a scintilletion vial containing $15 \mathrm{ml}$. of a scintillation mixture [60g. of naphthalene, $4 \mathrm{~g}$. of 2,5-diphenyloxazole, $0 \cdot 2 \mathrm{~g}$. of 1,4-bis-(5-phenyloxazol-2-yl)benzene, $100 \mathrm{ml}$. of methanol, $20 \mathrm{ml}$. of ethylene glycol and dioxan to 11.] to which Cab-O-Sil (Packard Instrument Co., Wembley, Middx.) had been added as a $5 \%$ suspension to prevent loss of radioactivity as a result of adsorption on the wall of the glass vial. The vials were counted in a Packard Tri-Carb liquid-scintillation counter (Packard Instrument Co.). No correction was applied for quenching.

[32P]G6P was counted by using the phosphate precipitation technique described for [ $\left.{ }^{32} \mathrm{P}\right] \mathrm{G} 1 \mathrm{P}$. After centrifugation, portions $(0.4 \mathrm{ml}$.) of the supernatants were added to scintillation vials containing scintillation mixture and Cab-O-Sil as in the determination of [ $\left.{ }^{32} \mathrm{P}\right] \mathrm{G} 1 \mathrm{P}$. Approx. $0.15 \mathrm{ml}$. of $\mathrm{N}-\mathrm{NaOH}$ was added to discharge the yellow colour and the vials were counted as described above.

$\left[{ }^{14} \mathrm{C}\right] \mathrm{GlP}$ was determined as glucose after hydrolysis and removal of G6P and G1P6P with $\mathrm{ZnSO}_{4}$ and $\mathrm{Ba}(\mathrm{OH})_{2}$. In experiments at low concentrations of substrates $0.1 \mathrm{ml}$. samples from the reaction mixture were each added to $0 \cdot 1 \mathrm{ml}$. of $\mathrm{N}_{-} \mathrm{H}_{2} \mathrm{SO}_{4}$ in glass-stoppered test tubes. The tubes were heated in a boiling-water bath for $10 \mathrm{~min}$. and, after cooling, $0.5 \mathrm{ml}$. of a solution containing glucose $(0.1 \mathrm{mg} . / \mathrm{ml}$.) was added to each followed by $0.65 \mathrm{ml}$. of $\mathrm{Ba}(\mathrm{OH})_{2}$ solution (approx. $\left.0 \cdot 15 \mathrm{M}\right)$. The tubes were agitated thoroughly, $0.2 \mathrm{ml}$. of $\mathrm{ZnSO}_{4}$ solution (approx. $5 \%, w / v$ ) was added to each and the tubes were again thoroughly agitated before the precipitate was compacted by centrifugation. Finally, $0 \cdot 1 \mathrm{ml}$. of the $\mathrm{Ba}(\mathrm{OH})_{2}$ solution followed by $0 \cdot 1 \mathrm{ml}$. of the $\mathrm{ZnSO}_{4}$ solution were added with thorough agitation. After centrifugation $0 \cdot 1-0 \cdot 2 \mathrm{ml}$. $(0 \cdot 7-1 \cdot 0 \mathrm{ml}$. in 
equilibrium-flux experiments) of each supernatant was pipetted into $15 \mathrm{ml}$. of dioxan scintillation medium in scintillation vials as in the determination of $\left[{ }^{32} \mathrm{P}\right] \mathrm{G} 1 \mathrm{P}$, but Cab-O-Sil was omitted. The vials were counted and corrected for quenching by the channels-ratio method.

The concentrations of $\mathrm{Ba}(\mathrm{OH})_{2}$ and $\mathrm{ZnSO}_{4}$ solutions were adjusted until $9 \cdot 8-9 \cdot 9 \mathrm{ml}$. of $\mathrm{Ba}(\mathrm{OH})_{2}$ solution added to $10 \mathrm{ml}$. of $\mathrm{ZnSO}_{4}$ solution (plus $5 \mathrm{ml}$. of water) produced a faint pink colour with phenolphthalein (Somogyi, 1945; Nelson, 1944). It was found that $6.4 \mathrm{ml}$. of this $\mathrm{Ba}(\mathrm{OH})_{2}$ solution also gave a faint pink colour with phenolphthalein when added to $2 \mathrm{ml}$. of $\mathrm{ZnSO}_{4}$ solution and $1 \mathrm{ml}$. of $\mathrm{N}-\mathrm{H}_{2} \mathrm{SO}_{4}$. There was thus a very slight excess of $\mathrm{Ba}^{2+}$ at the end of each of the precipitation steps.

The procedure outlined above was suitable for experiments in which the total substrate concentration did not exceed approx. 5mm. For higher concentrations the volumes were increased. At $30.4 \mathrm{~mm}$ total concentration (Fig. 2) the volumes of reagents were doubled and the sample volume was decreased to $0.05 \mathrm{ml}$. At $86.4 \mathrm{~mm}$ total concentration (Fig. 3) the reagent volumes were increased 20-fold, apart from the final addition of $\mathrm{ZnSO}_{4}$ and $\mathrm{Ba}(\mathrm{OH})_{2}$, where $1 \mathrm{ml}$. of each solution was added; $2 \mathrm{ml}$. of the supernatant was taken for counting.

Total radioactivity in the reaction mixtures was determined by taking $0.1 \mathrm{ml}$. samples and adding to $15 \mathrm{ml}$. of scintillation mixture containing Cab-O-Sil.

$\left[{ }^{14} \mathrm{C}\right]$ G1P6P was determined as follows. Samples $(0 \cdot 1 \mathrm{ml}$.) taken from the reaction mixture were heated very briefly at $100^{\circ}$ and applied to DEAE-cellulose chromatography paper (Whatman DE81). Descending chromatography was then carried out for $6 \mathrm{hr}$. with $0 \cdot 18 \mathrm{M}$-ammonium formate and the chromatogram was scanned with a Packard scanner (Packard Instrument Co.).

Induced-transport tests. The enzyme was activated in histidine-tris buffer as described under 'Reagents and materials' above and suitably diluted with the same buffer. G1P and $\left[{ }^{32} \mathrm{P}\right] \mathrm{GlP}$, or $\mathrm{G6P}$ and $\left[{ }^{14} \mathrm{C}\right] \mathrm{G} 6 \mathrm{P}$, and $\mathrm{GlP} 6 \mathrm{P}$ were then added and the system was incubated for $60-90 \mathrm{~min}$. at $30^{\circ}$ to allow it to come into equilibrium. Non-radioactive G1P was then added in small volume to the reaction mixture. Samples $(0 \cdot 1 \mathrm{ml}$.) were taken for chemical and radioactivity determinations before and after the addition of nonradioactive G1P. In one series of experiments ( $A$ and $C$ in Fig. 1 and Figs. 4 and 5), $4 \mathrm{ml}$. of the enzyme solution was taken and $1 \mathrm{ml}$. of a solution containing G1P, or G6P, and G1P6P was added; the non-radioactive G1P was added in a volume of $0.4 \mathrm{ml}$. to $4.6 \mathrm{ml}$. of this solution. The concentrations of histidine, tris and $\mathrm{MgCl}_{2}$ were $29 \cdot 4,8 \cdot 8$ and $0 \cdot 8 \mathrm{mM}$ respectively after the addition of the non-radioactive G1P. In the other series of experiments $(B$ in Fig. 1 and Figs. 2 and 3), $1.35 \mathrm{ml}$. of a solution containing G6P and G1P6P was added to $4 \mathrm{ml}$. of the enzyme solution; the non-radioactive G1P was added in a volume of $0.4 \mathrm{ml}$. to $4.95 \mathrm{ml}$. of this solution. In this case the final concentrations of histidine, tris and $\mathrm{MgCl}_{2}$ were $27 \cdot 7,8 \cdot 3$ and $0 \cdot 76 \mathrm{~mm}$ respectively.

When $\left[{ }^{14} \mathrm{C}\right] \mathrm{G} 1 \mathrm{P}$ was determined it was necessary to allow for radioactivity due to incomplete precipitation of G6P by $\mathrm{ZnSO}_{4}$ and $\mathrm{Ba}(\mathrm{OH})_{2}$, partial hydrolysis of $\mathrm{G6P}$ and radioactive impurities in the $\left[{ }^{14} \mathrm{C}\right] \mathrm{G} 6 \mathrm{P}$. Of these the last appeared to be the most important. Radioactivity from these sources, which amounted to about $10 \%$ of the total, was determined by carrying out a blank experiment in which no enzyme was present. When allowance was made for this the radioactivity in the G1P agreed with that calculated to be present from the equilibrium constant.

When [ $\left.{ }^{32} \mathrm{P}\right] \mathrm{GlP}$ was determined allowance had to be made, not only for partial hydrolysis of $\mathrm{G} 6 \mathrm{P}$ and radioactive impurities of the GIP, but for the contribution from the phosphate derived from the 1-position of G1P6P. A correction factor was derived by subtracting the radioactivity, calculated to be present in the GIP from the total radioactivity added and the equilibrium constant, from the radioactivity found in the G1P during the equilibrium period. The correction factor usually amounted to about 15-20\% of the total radioactivity found in the G1P. An equilibrium constant of $1 / 17 \cdot 2$ was assumed (Colowick \& Sutherland, 1942; Ray \& Roscelli, 1964b); the chemical determinations of GIP in the present paper yielded equilibrium constants within $2 \%$ of this value at all concentrations.

Comparison of glucose and phosphate fluxes at chemical equilibrium. A solution of the enzyme in histidine-tris buffer was prepared and activated as described under 'Reagents and materials' above. G1P and G1P6P were added as in the induced-transport experiments $(0.42 \mathrm{ml}$. to $7.78 \mathrm{ml}$. of enzyme-buffer solution) and the system was allowed to come into equilibrium at $30^{\circ}$. The solution was divided into two equal $4 \mathrm{ml}$. portions and [ $\left.{ }^{32} \mathrm{P}\right] \mathrm{GlP}$ and $\left[{ }^{14} \mathrm{C}\right] \mathrm{G6P}$ were added respectively to the two portions in small volume $(0 \cdot 01-0 \cdot 025 \mathrm{ml}$.). Samples $(0 \cdot 1 \mathrm{ml}$.) were then taken at intervals for measurement of radioactivity. The concentrations of histidine, tris and $\mathrm{MgCl}_{2}$ were $37 \cdot 5,11 \cdot 2$ and $1.03 \mathrm{~mm}$ in the final reaction mixture.

To calculate the fluxes the logarithms to base 10 of the differences between the actual counts in individual samples and the final equilibrium value were plotted against time and the best straight line was drawn. The fluxes were derived from the slope of the line by using the expression:

$$
\text { Flux }=\frac{2 \cdot 303 V}{\left(\frac{1}{[\mathrm{GlP}]}+\frac{1}{[\mathrm{G} 6 \mathrm{P}]}\right)(\mathrm{E})} \text { (Slope) }
$$

where $V$ is the volume of solution, (E) is the total quantity of enzyme and [G1P] and [G6P] represent concentrations. No allowance was made for radioactivity appearing in the G1P6P, since Ray et al. (1966) found a negligible transfer of radioactivity into this material. Further, the concentration of the G1P6P was only about $5 \%$ of the G1P concentration.

Depression of freezing point. Freezing-point determina-

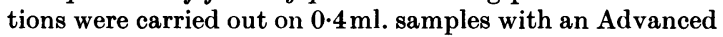
osmometer (Advanced Instruments Inc., Newton Highlands, Mass., U.S.A.).

\section{RESULTS}

In all the experiments the reaction was carried out in a histidine-tris buffer, $\mathrm{pH} 7 \cdot 4$, at $30^{\circ}$ with activated enzyme (Ray \& Roscelli, 1964a) and in the presence of excess of G1P6P. In considering the concentration of G1P6P competition from G1P and G6P had to be taken into account, but calculations from the data of Ray \& Roscelli $(1964 a, b)$ indicated that concentration of G1P6P exceeded the effective $K_{m}$ for G1P6P by five- to ten-fold. 
Induced-transport tests with ${ }^{14} \mathrm{C}$-labelled substrates. The results of a series of induced-transport tests at different substrate concentrations with ${ }^{14} \mathrm{C}$ labelled substrates are illustrated in Figs. 1-3. Where single experiments only are shown the results have been confirmed by duplicate experiments that are not illustrated. At low concentrations (Fig. 1) the radioactivity in the G1P remained substantially unchanged during the reaction, but at the concentrations used in Fig. 2 there was probably a small rise in the radioactivity, and at the high concentrations in Fig. 3 there was a rise of about $10 \%$ while the reaction was in progress.

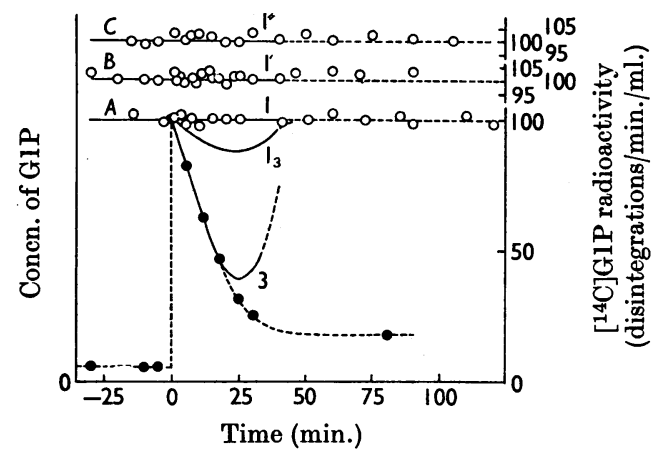

Fig. 1. Induced-transport tests at low substrate concentrations with ${ }^{14} \mathrm{C}$-labelled substrates showing absence of induced transport. $\left[{ }^{14} \mathrm{C}\right] \mathrm{G} 6 \mathrm{P}$ was incubated at $30^{\circ}$ with rabbit muscle phosphoglucomutase in a histidine-tris buffer, $\mathrm{pH} 7 \cdot 4$, containing G1P6P until equilibrium was reached. Samples were then taken from the reaction mixture and at zero time non-radioactive G1P was added. ๑, Concn. of G1P; O, [14C]G1P radioactivity (disintegrations $/ \mathrm{min} . / \mathrm{ml}$.) corrected for dilution caused by addition of G1P. $A$, Non-radioactive G1P was added in a volume of $0.4 \mathrm{ml}$. to $4.6 \mathrm{ml}$. of solution. Concentrations before addition: G1P, 0.0404 mM; G6P, 0.695 mM ; G1P6P, $2 \cdot 13 \mu \mathrm{M}$; concentrations immediately after addition: G1P, $1.044 \mathrm{mM}$; G6P, 0.630 mM; G1P6P, 2.0 $\mu$ M. $B$, Non-radioactive G1P was added in a volume of $0.4 \mathrm{ml}$. to $4.95 \mathrm{ml}$. of solution. Concentrations before addition: G1P, 0.087 mM; G6P, $1.51 \mathrm{mM}$; G1P6P, 4.6 $\mu \mathrm{M}$; concentrations immediately after addition: G1P, 1.58mM; G6P, $1.39 \mathrm{~mm}$; G1P6P, $4.2 \mu \mathrm{M}$. The points shown represent means of duplicate or triplicate observations. $C$, Non-radioactive G1P was added in a volume of $0.4 \mathrm{ml}$. to $4 \cdot 6 \mathrm{ml}$. of solution. Concentrations before addition: G1P, 0.0808mm; G6P, 1.39mM; G1P6P, $4.26 \mu \mathrm{M}$; concentrations immediately after addition : G1P, 2.088mM; G6P, 1.26 mM; G1P6P, 4.0 $\mu \mathrm{M}$. In individual experiments the time-course of the reaction varied slightly. The time-scale of each experiment was therefore adjusted so that the course of the reaction was represented by the curve shown. The continuous lines are theoretical curves: $1,1^{\prime}$ and $1^{\prime \prime}$ for mechanism 1 or mechanism 2, assuming $\alpha=0.637 \times 10^{-2} \mathrm{~mm}^{-1} ; 3$, for mechanism $3 ; 1_{3}$, for $80 \%$ of the glucose flux from G1P to G6P proceeding by mechanism 1 or mechanism 2 and $20 \%$ proceeding by mechanism 3.
The increase in radioactivity in Fig. 3 appeared to be significant, since it occurred in all experiments, and no rise took place in control experiments in which the enzyme was inactivated by brief heating immediately before the addition of non-radioactive G1P. At the end of the reaction the radioactivity in the G1P always returned substantially to the value existing before the addition of non-radioactive G1P.

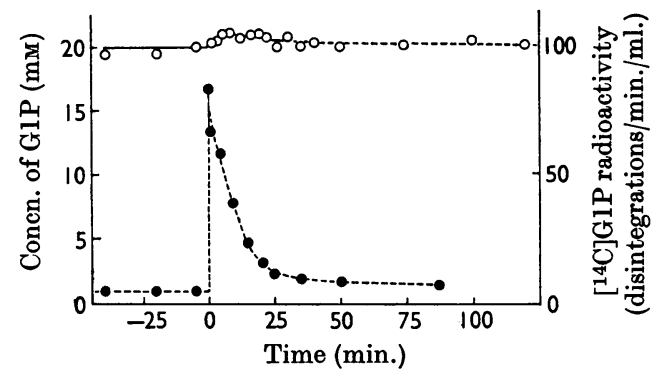

Fig. 2. Induced-transport test with ${ }^{14} \mathrm{C}$-labelled substrates at intermediate substrate concentrations. For symbols and experimental procedure see the legend to Fig. 1. At zero time non-radioactive G1P in $0.4 \mathrm{ml}$. was added to $4.95 \mathrm{ml}$. of reaction mixture. Concentrations before addition of non-radioactive G1P: G1P, $0.869 \mathrm{mM}$; G6P, 14.92 mM; G1P6P, 0.0489 mM; concentrations immediately after addition: G1P, 16.53 mM; G6P, 13.74mM; G1P6P, 0.0450mM. The continuous line is the theoretical curve for mechanism 1 or mechanism 2 assuming $\alpha=0.637 \times 10^{-2} \mathrm{~mm}^{-1}$.

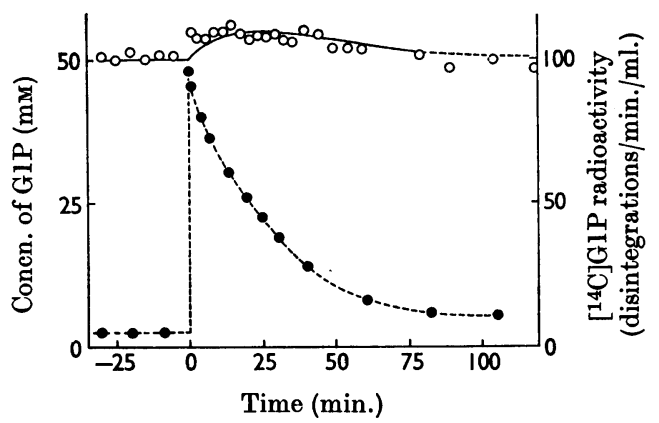

Fig. 3. Induced-transport test at high substrate concentrations with ${ }^{14} \mathrm{C}$-labelled substrates showing counter-transport. For symbols and experimental procedure see the legend to Fig. 1. The points shown represent means of duplicate or triplicate determinations. At zero time non-radioactive G1P in $0.4 \mathrm{ml}$. was added to $4.95 \mathrm{ml}$. of reaction mixture. Concentrations before addition: GIP, 2.465 mM; G6P, 42.4 mM; GIP6P, $0.1155 \mathrm{mM}$; concentrations immediately after addition: G1P, 47.1 mM; G6P, 39.2 mM; G1P6P, $0 \cdot 1059 \mathrm{~mm}$. The continuous line is the theoretical curve for mechanism 1 or mechanism 2 assuming $\alpha=0.637 \times 10^{-2} \mathrm{~mm}^{-1}$. 
It is possible that after the addition of nonradioactive G1P (Figs. 1-3) some radioactivity was transferred from the G1P6P to the G1P, but (see below) any contribution from this source must have been very small. Consequently a change in the radioactivity in the G1P must very largely reflect a corresponding change in the radioactivity in the G6P. Thus the experiments shown in Figs. 1-3 provide strong evidence for a lack of induced transport of ${ }^{14} \mathrm{C}$-labelled substrates at low substrate concentrations and counter-transport at high concentrations. If isotope effects and non-ideal behaviour of solutions, which are probably relatively unimportant (see below), are ignored, it follows that the glucose fluxes must obey the Independence relationship at low substrate concentrations, but that at high concentrations the flux ratio must deviate from this relationship in the direction of exchange diffusion. Such a result is clearly compatible with either mechanism 1 or mechanism 2, for which the equations are identical, and the continuous lines drawn through the experimental points in Figs. 1-3 are the theoretical lines for $\alpha=0.637 \times 10^{-2} \mathrm{~mm}^{-1}$ (eqn. 9). Exchange diffusion provides evidence that there is a rate-limiting isomerization of the phosphoenzyme, and if nonideal behaviour of the solution is ignored the apparent rate constant for the isomerization step (if $k_{+3}=k_{-3}$ ) will be about $4.5 \times 10^{7} \mathrm{sec}^{-1}$, as is shown in the Appendix.

The results in Figs. 1-3 exclude mechanism 3, since for this mechanism the glucose fluxes should show the chemical analogue of the long-pore effect at low concentrations of substrate and co-transport should have occurred. Thus the theoretical curves 3 and $1_{3}$ in Fig. 1 show the calculated results if the whole reaction had proceeded via mechanism 3 or if $20 \%$ of the flux from G1P to G6P had proceeded via mechanism 3 , assuming that $\alpha$ (eqn. 19) is small, and the theoretical curves clearly diverge from the experimental results. If the value of $\alpha$ (eqn. 19) were large the predicted amount of co-transport would be less and the theoretical lines would be closer to the experimental results. However, if this were the case, the net reaction should have shown product inhibition of a partly non-competitive nature, and Ray \& Roscelli (1964b) were unable to detect any inhibition of this kind. Thus it may be concluded that the experimental results exclude even a small contribution of mechanism 3 to the reaction.

As mentioned above, the possibility that radioactivity was transferred transiently from the G1P6P to the G1P when the non-radioactive G1P was added has to be considered, because of the low specific radioactivity of the G1P immediately after the addition. To test for this possibility samples were taken and chromatographed on DEAE- cellulose paper with ammonium formate to separate the G1P6P from the monophosphates. The chromatograms were then scanned for radioactivity. The G1P6P had the same specific radioactivity as the substrates at the end of the equilibration period, and when the non-radioactive G1P was added the specific radioactivity fell progressively to reach the expected value of about one-half the initial radioactivity. However, at the time when the radioactivity in the GIP was elevated in Fig. 3, the radioactivity in the G1P6P had fallen by less than $20 \%$. The total radioactivity in the GIP6P at the end of the equilibration period was only about $5 \%$ at the lower concentrations, and $2.5 \%$ at the highest concentrations of the radioactivity in the G1P. Thus the G1P6P could have contributed at most only $0.5-1 \%$ to the radioactivity in the G1P. Further, it is extremely unlikely that all the radioactivity disappearing from the G1P6P would be transferred to the G1P. For example, by using the rate constants obtained by Ray \& Roscelli (1964a,b, 1966a) it may be calculated that only about one-quarter of the radioactivity would be so transferred. However, the latter calculation may not be valid, since Ray \& Roscelli $(1964 a, b, 1966 a, b)$ in their analysis assumed only one intermediate enzymesubstrate complex, and there is evidence suggesting that there may be at least two complexes and that G1P6P can dissociate from both of them. Thus from initial-rate studies with G1P Ray \& Roscelli $(1964 a, b)$ obtained an apparent $K_{m}$ for G1P6P of $6.2 \times 10^{-8} \mathrm{M}$, whereas they obtained a value of $1 \cdot 1( \pm 0 \cdot 5) \times 10^{-8} \mathrm{M}$ from initial-rate studies with G6P under similar experimental conditions. Such a difference could be most simply explained by assuming two intermediate enzyme-substrate complexes. However, it seems that the G1P6P must have contributed very little radioactivity to the G1P during the reaction and that any radioactivity appearing in the G1P must have been largely derived from the G6P. At the end of the reaction there will be a small contribution from the G1P6P to the G1P, but this will be less than $0.1 \%$ of the initial radioactivity.

Ray \& Roscelli (1964a), using 32P-labelled phosphoenzyme, concluded that the enzymesubstrate complex dissociated into G1P6P on about $5 \%$ of occasions, but the present observations suggest that the frequency of dissociation must be rather smaller. Hashimoto \& Handler (1966) studied the rate of incorporation of ${ }^{32} \mathrm{P}$-labelled phosphate into G1P6P with [ $\left.{ }^{32} \mathrm{P}\right] \mathrm{G} 1 \mathrm{P}$ as substrate and also obtained results that indicated a low rate of dissociation of the enzyme-substrate complex. Further, in later experiments Ray et al. (1966) found negligible incorporation of radioactivity into G1P6P in equilibrium flux measurements with 32P-labelled substrates. It is therefore 
possible that in the original experiments of Ray \& Roscelli (1964a) the frequency of dissociation was overestimated. This could have arisen if there were some [32P]G1P6P rather tightly bound to the phosphoenzyme; alternatively, there may have been some alteration of the enzyme during the mixing procedure, perhaps as a result of transient electrolyte changes. The latter might also explain the relatively rapid incorporation of 32P-labelled phosphate into G1P6P that occurred in the initial stages of the experiments of Hashimoto \& Handler (1966). That such enzyme changes may occur is suggested by the work of Cascales \& Grisolia (1966) on glycerophosphomutase in which the rate of incorporation of ${ }^{32} \mathrm{P}$-labelled phosphate into diphosphoglyceric acid from the substrates was greatly increased when the ionic strength of the solution was raised.

Induced-transport tests with ${ }^{32} \mathrm{P}$-labelled substrates. Induced-transport tests with ${ }^{32} \mathrm{P}$-labelled substrates are illustrated in Figs. 4 and 5. In Fig. 4 the results of a single experiment are shown in which the radioactivity in the G1P fell after the addition of non-radioactive G1P and the initial rate of fall was similar to the rate of chemical reaction. Therefore under these conditions the net flux must have been virtually equal to the flux of [ $\left.{ }^{32} \mathrm{P}\right] \mathrm{G} 1 \mathrm{P}$ to G6P, and the flux of [32P] G6P to G1P must have been very small. As the reaction progressed, however, the two curves diverged, and the radioactivity in the G1P reached a minimum of $39 \%$ and then returned to its initial value. Fig. 4 also shows for comparison the results of the comparable experiment with ${ }^{14} \mathrm{C}$-labelled substrates. A second experiment under the conditions shown in Fig. 4 yielded a very similar result with the same minimum of $39 \%$ for the radioactivity in the GIP. In Fig. 5 the experimental conditions were similar to those in Fig. 4, but the concentrations of substrates were doubled. Again a very similar result was obtained. The minima for the radioactivity in the G1P for the two experiments from which the data shown in Fig. 5 were obtained were 37 and $40 \%$.

Clearly in these experiments considerable cotransport of the ${ }^{32} \mathrm{P}$-labelled substrates occurred, but, as in the experiments with ${ }^{14} \mathrm{C}$-labelled substrates, the contribution from the G1P6P to the radioactivity measured in the GlP has to be considered. This is discussed below, but the effect is slight and amounts to a probable overestimate of the amount of co-transport by rather less than $1 \%$ of the initial radioactivity in the G1P. The fact that co-transport occurred indicates that the phosphate flux ratio must show the chemical analogue of the long-pore effect. This observation therefore qualitatively supports either mechanism 1 or mechan. ism 3 and excludes mechanism 2 . It is possible to calculate quantitatively the amount of co-transport

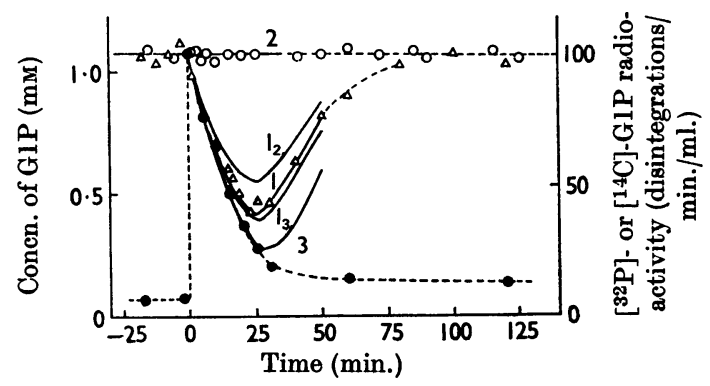

Fig. 4. Induced-transport test with ${ }^{32} \mathrm{P}$-labelled substrates showing co-transport. [ $\left.{ }^{32} \mathrm{P}\right]$ G1P was incubated at $30^{\circ}$ with rabbit muscle phosphoglucomutase in a histidine-tris buffer, pH7.4, until equilibrium was reached. Samples were then taken from the reaction mixture and at zero time non-radioactive GlP was added in a volume of $0.4 \mathrm{ml}$. to $4.6 \mathrm{ml}$. of the mixture. $\bullet$, Concn. of G1P; $\Delta,\left[{ }^{32} \mathrm{P}\right] \mathrm{G} 1 \mathrm{P}$ radioactivity (disintegrations/min./ml.) corrected for dilution caused by addition of non-radioactive G1P; $O,\left[{ }^{14} \mathrm{C}\right] \mathrm{G} 1 \mathrm{P}$ radioactivity (disintegrations/min./ml.) of comparable experiment taken from Fig. 1. Concentrations before addition: G1P, 0.0404 mM ; G6P, 0.695 mM; G1P6P, $2 \cdot 13 \mu \mathrm{M}$. Concentrations immediately after addition: G1P, $1.044 \mathrm{mM}$; G6P, $0.630 \mathrm{mM}$; G1P6P, $2 \cdot 0 \mu \mathrm{M}$. The continuous lines are theoretical curves for ${ }^{32} \mathrm{P}$-labelled substrates: 1 , for mechanism 1 ; 2, for mechanism 2; 3, for mechanism 3; $1_{2}$, for $80 \%$ of flux of G1P to G6P proceeding by mechanism 1 and $20 \%$ proceeding by mechanism $2 ; 1_{3}$, for $80 \%$ of flux from G1P to G6P proceeding by mechanism 1 and $20 \%$ proceeding by mechanism 3.

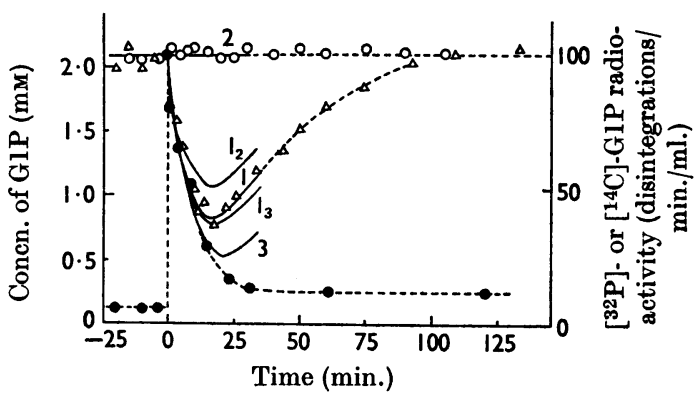

Fig. 5. Induced-transport tests with ${ }^{32}$ P-labelled substrates to show that the co-transport is quantitatively unaffected by a twofold increase in substrate concentration. For symbols and experimental procedure see the legend to Fig. 4. The points shown represent means of duplicate observations. Concentrations before addition: G1P, $0.0808 \mathrm{~mm}$; G6P, 1.39 mM ; G1P6P, $4.26 \mu \mathrm{M}$. Concentrations immediately after addition : G1P, $2.088 \mathrm{mM}$; G6P, $1.26 \mathrm{mM}$; G1P6P, 4.0 $\mu \mathrm{M}$.

for the different mechanisms and for various combinations of them (see the Appendix). The theoretical curves, which are the same at both concentrations, are shown as continuous lines in Figs. 4 and 5. 
The experimental results correspond closely to the curve for mechanism 1 , and the theoretical minimum for the radioactivity in the G1P of $39 \cdot 1 \%$ agrees well with the minima for the radioactivity in the individual experiments. The curves for mechanisms 2 and 3 differ widely from the experimental results, so that these mechanisms can be excluded as major reaction pathways. The curve for $20 \%$ of the flux from G1P to G6P proceeding via mechanism 2 and $80 \%$ proceeding via mechanism 1 also differs considerably from the experimental results, so that the possibility of any substantial but minor contribution from mechanism 2 is also excluded. The results do not exclude the possibility of a small part of the reaction proceeding via mechanism 3; however, this has already been excluded by the induced-transport tests with ${ }^{14} \mathrm{C}$-labelled substrates.

To calculate the induced-transport curves $\alpha$ (eqns. 8 and 18) has been assumed to be small and of the order of that found in the induced-transport tests with ${ }^{14} \mathrm{C}$-labelled substrates. The justification for this assumption has already been discussed above in relation to these experiments.

In the above, isotope effects and non-ideal behaviour of the solutions have been ignored, but these effects should be unimportant (see below).

To consider the contribution from the G1P6P to the radioactivity found in the G1P it should be remembered that the method of estimation of G1P also determined the phosphate in the 1-position of the G1P6P. An allowance was made for the radioactivity contributed by the G1P6P by comparing the radioactivity found in the GIP, at the end of the equilibration period, with the radioactivity calculated to be present in this substrate (see the Materials and Methods section). This correction will be valid after the addition of non-radioactive G1P only if the amount of radioactivity in the 1-position of the G1P6P remains constant. Immediately after the addition of non-radioactive G1P, the enzymesubstrate complex was most frequently formed by reaction of the phosphoenzyme with G1P, and since the specific radioactivity of the G1P fell by a large factor the enzyme-substrate complex and any G1P6P formed by dissociation of this complex subsequent to the addition must have had little radioactivity in the 1-position. However, from the chromatographic results obtained in the experiments with ${ }^{14} \mathrm{C}$-labelled substrates not more, and probably less, than $20 \%$ of the G1P6P present at the time of the minima in Figs. 4 and 5 had been formed after the addition of the non-radioactive G1P. Thus, as the phosphate in the 1-position of the G1P6P represented about $5 \%$ of the total acidlabile phosphate, it follows that the fall in the amount of labelling in the 1-position of the G1P6P must have represented not more than $1 \%$ of the initial radioactivity in the GlP. A proportion of the radioactivity lost from the 1-position of the G1P6P must have been transferred to the G1P, as has been discussed above in relation to the results obtained with ${ }^{14} \mathrm{C}$-labelled substrates, and this part would not have contributed to the error.

Comparison of glucose and phosphate fluxes at chemical equilibrium. The results of equilibrium flux-ratio measurements at two different substrate concentrations are shown in Table 1. The ratio of the glucose flux to the phosphate flux determined with ${ }^{14} \mathrm{C}$ - and ${ }^{32} \mathrm{P}$-labelled substrates was close to $2: 1$ at both concentrations. This result excludes mechanism 2, which would have given a 1:1 ratio, but is in accord with mechanism 1 provided that $\alpha$ is small, as was indicated by the induced-transport experiments with ${ }^{14} \mathrm{C}$-labelled substrates. For mechanism 3 the flux ratio would be $3: 2$ if $\alpha$ were small, but would approach $2: 1$ if $\alpha$ were large. However, the latter possibility is excluded, since Ray \& Roscelli (1964b) could not detect any product inhibition that was not strictly competitive. Thus the equilibrium flux ratios are only compatible with mechanism 1 as the major pathway. If a minor part of the reaction proceeded by mechanism 2 or mechanism 3 the effect would be to decrease the flux ratio, but the equilibrium flux ratio is not a very sensitive test for such pathways. Thus if $20 \%$ of the glucose flux proceeded by mechanism 2 or

Table 1. Fluxes of glucose and phosphate at chemical equilibrium determined by the use of ${ }^{14} \mathrm{C}$ - and ${ }^{32} \mathrm{P}$-labelled substrates

The measurements were made in a histidine-tris buffer, $\mathrm{pH} 7 \cdot 4$, at $30^{\circ}$.

\begin{tabular}{|c|c|}
\hline \multicolumn{2}{|c|}{$\begin{array}{l}\text { Concn. of hexose } \\
\text { phosphates } \\
(\mathrm{mm})\end{array}$} \\
\hline G1P & 0.055 \\
\hline G6P & 0.945 \\
\hline G1P6P & 0.003 \\
\hline GlP & $0 \cdot 138$ \\
\hline G6P & $2 \cdot 362$ \\
\hline G1P6P & 0.0077 \\
\hline
\end{tabular}

$\begin{gathered}\text { Phosphate flux } / \\ \mu \mathrm{g} . \text { of enzyme } \\ (\mu \mathrm{moles} / \mathrm{min} . / \mu \mathrm{g} .)\end{gathered}$
$12 \cdot 0$
$17 \cdot 3$
$17 \cdot 6$
$23 \cdot 6$

Phosphate flux/ $\mu \mathrm{g}$. of enzyme ( $\mu$ moles/min./ $\mu \mathrm{g}$. ( $\mu \mathrm{g} . / \mathrm{ml}$.

$0 \cdot 686$

$1 \cdot 65$ 
mechanism 3 the flux ratios would become 1.8:1 and 1.9:1 respectively, assuming $\alpha$ to be small as is required by the lack of non-competitive product inhibition.

Summary of results. The induced-transport tests with ${ }^{14} \mathrm{C}$-labelled substrates were compatible with mechanisms 1 and 2, but excluded even a minor contribution from mechanism 3. The inducedtransport tests with ${ }^{32} \mathrm{P}$-labelled substrates, on the other hand, gave the expected result for mechanism 1 and excluded any contribution from mechanism 2; any large contribution from mechanism 3 was also excluded. The equilibrium flux-ratio measurements again indicated that mechanism 1 must be the only major pathway. Thus, of the three, mechanism 1 appears to be the only possible mechanism for the reaction. The occurrence of counter-transport with ${ }^{14} \mathrm{C}$-labelled substrates at high concentrations provides, in addition, evidence for a rate-limiting isomerization of the phosphoenzyme.

\section{DISCUSSION}

In the foregoing, the influence of isotope effects and non-ideal behaviour of the solutions has been ignored and the results have been discussed in relation to particular models only. These factors and the more general significance of the results are now considered. Induced-transport tests and equilibrium flux-ratio measurements are discussed separately.

Induced-transport tests. (a) Isotope effects and non-ideal behaviour of solutions. In the present work the absence of induced transport has been used as evidence for the flux ratio obeying the Independence relationship (eqn. 21) and the magnitude of any induced transport has been used as a measure of the deviation of the flux ratio from this relationship. However, it is shown in the Appendix that, in fact, there will be an absence of induced transport when the flux ratio is given by Appendix eqn. (10) and the magnitude of any induced transport will be given by the integral of Appendix eqn. (21). The relationship between these equations and eqn. (21) is discussed in the Appendix and it is concluded that lack of induced transport must necessarily be evidence for the reaction obeying the Independence relationship even if isotope effects are present and the solutions behave non-ideally, provided that the reaction has only one pathway and provided that the ratio of the activity coefficients for the labelled substrates is unaffected by the addition of unlabelled substrate. In contrast, the magnitude of any induced transport will be influenced by isotope effects and may be also affected by the activity coefficients. When there are alternative pathways for the reaction lack of induced transport will not necessarily be evidence for the reaction obeying the Independence relationship, since the isotope may preferentially follow one pathway, but the induced-transport test may still provide evidence for steps in the reaction (see the Appendix).

Unless the isotopically labelled substrate followed one pathway to the exclusion of other pathways, the present results provide strong evidence that the reaction proceeds by only one pathway. Isoenzymes with similar reaction pathways (see below), and the very small part of the reaction that involves the substrates adding to the enzyme before the attachment of $\mathrm{Mg}^{2+}$ (Ray \& Roscelli, 1966b; Ray et al. 1966), do not constitute alternative pathways for present purposes. Thus, if activity coefficients are ignored, the lack of induced transport of ${ }^{14} \mathrm{C}$-labelled substrates at low substrate concentrations must be good evidence for the reaction obeying the Independence relationship even if isotope effects are present. The counter-transport that was seen at high substrate concentrations is sensitive to isotope effects, but such effects would seem to be unimportant in relation to the other uncertainties of measurement. In the induced-transport experiments with ${ }^{32} \mathrm{P}$ labelled substrates isotope effects would be important, since the magnitude of the co-transport was used to distinguish between the mechanisms. However, with 32P-labelled substrates isotope effects should be very small, and the experiments themselves provided some evidence that such effects were not significant. Thus the initial rates of fall of G1P concentration and $\left[{ }^{32} \mathrm{P}\right] \mathrm{GlP}$ radioactivity, after the addition of non-radioactive G1P, in Figs. 4 and 5, were equal. If an isotope effect had been present the rate of fall of [ $\left.{ }^{32} \mathrm{P}\right]$ G1Pradioactivity might have been expected to be less rapid than the fall in G1P concentration.

As mentioned above, lack of induced transport can only be used as a criterion for the reaction obeying the Independence relationship if the ratio of the activity coefficients for the labelled substrates is uninfluenced by the addition of unlabelled substrate. The ratio of the activity coefficients for the labelled species should be similar to the ratio for the unlabelled substrates; the fact that the equilibrium constant was the same for both ${ }^{14} \mathrm{C}$ labelled and unlabelled substrates provides experimental support for this assumption (see the Materials and Methods section). Thus it would seem sufficient to show that the ratio for the unlabelled substrates is unchanged when additional substrate is added in order to assume that the ratio for the labelled substrates remains constant. In the present case there is experimental evidence (see below) that the ratio for the unlabelled substrates is constant and close to unity. Lack of induced transport will thus be evidence for the reaction obeying the Independence relationship. Whether the 
magnitude of any induced transport is also independent of the activity coefficients under these conditions depends on the nature of the interaction between the fluxes. The magnitude will be independent of the activity coefficients if the interaction between the fluxes is due to an indirect transfer of groups (as, for example, is the case with phosphate in mechanisms 1 and 3), but not if the induced transport is caused by an interaction due to an isomerizing step (see Appendix eqn. 21).

The properties of G1P and G6P are very similar and the ratio of their activity coefficients may be expected to be close to $1: 1$. Thus G1P and G6P have very nearly the same ionic dissociation constants (Cori, Colowick \& Cori, 1937) and electrostatic effects should influence both equally; binding by $\mathrm{Mg}^{2+}$ should be small (Ray et al. 1966) and may be expected to be similar for both; and the similarity between the molecules suggests that non-ionic factors will influence both in the same way. Evidence that the ratio of the activity coefficients is $1: 1$ is given by the fact that the equilibrium constant was independent of $\mathrm{pH}$ (Colowick \& Sutherland, 1942) and by the fact that the equilibrium constant was independent of the concentration of the reactants, within $2 \%$, in the present work (see the Materials and Method section). In addition, experiments on depression of freezing point, which are illustrated in Fig. 6, showed that G1P and G6P gave virtually the same freezing-point depression despite the fact that the activity coefficients fell substantially at the higher concentrations.

If the counter-transport of ${ }^{14} \mathrm{C}$-labelled substrates at high concentrations (Fig.3) had been due to the ratio of the activity coefficients departing from $1: 1$ the departure would have had to be relatively large, since the theoretical curve in Fig. 3 requires a $30 \%$ deviation of the flux ratio from Appendix eqn. (10) immediately after the addition of non-radioactive G1P. Perhaps the only way in which this could occur would be if the G1P molecules interacted with each other, for example with the formation of dimers. This possibility appears to be excluded by the freezing-point data, especially since shortly after the addition of the non-radioactive GlP the concentrations of G1P and G6P became the same, and a direct comparison of the freezing-point data over the same range of concentrations becomes possible. Further, dimer formation of the required order of magnitude should alter the equilibrium constant by about $7.5 \%$ at the end of the reaction, whereas in fact no change was observed. Indeed, the constancy of the equilibrium constant and the freezing-point data taken together suggest that neither G1P or G6P associates in solution.

(b) Significance of induced-transport tests with ${ }^{14} \mathrm{C}$-labelled substrates. From the above it is seen that the virtual absence of induced transport of

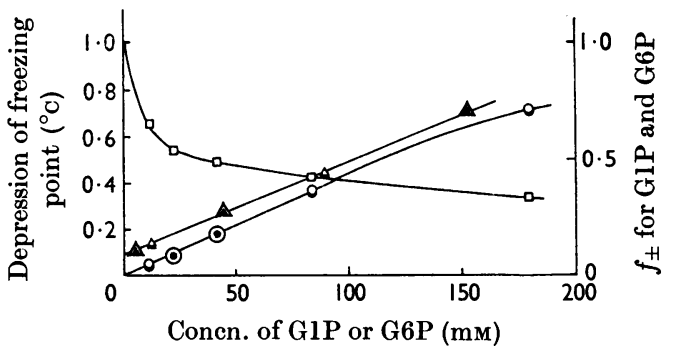

Fig. 6. Depression of freezing point of solutions of G1P and G6P. O and $\bullet, \mathrm{Na}_{2} \mathrm{GlP}$ and $\mathrm{Na}_{2} \mathrm{G} 6 \mathrm{P}$ in water; $\triangle$ and $\Delta$, $\mathrm{Na}_{2} \mathrm{GlP}$ and $\mathrm{Na}_{2} \mathrm{G} 6 \mathrm{P}$ in a histidine-tris buffer, $\mathrm{pH} 7 \cdot 4$ (histidine, 32 mM; tris, 9.6 mM ; $\mathrm{MgCl}_{2}, 0.88 \mathrm{~mm}$ ). $\square$, Mean ionic activity coefficients of G1P and G6P at $0^{\circ}$ calculated from the data for $\mathrm{Na}_{2} \mathrm{GlP}$ and $\mathrm{Na}_{2} \mathrm{G} 6 \mathrm{P}$ in water (for details of the calculation see the Appendix). Equimolar mixtures of G1P and G6P gave freezing-point depressions that were the means of the results for the corresponding solutions of G1P and G6P of the same total molarity.

${ }^{14} \mathrm{C}$-labelled substrates over a nearly 30-fold concentration range must constitute very good evidence for the glucose fluxes obeying the Independence relationship (eqn. 21) over this range of concentrations. A reaction obeying the Independence relationship must either possess no steps that bring about an interaction between the fluxes, or if such steps exist the interactions they bring about must effectively cancel each other. The latter possibility would be excluded if it could be demonstrated that the Independence relationship was obeyed over a wide range of concentrations, since cancellation would be expected only over a limited range. Such an observation would be good evidence for a direct transfer of substrate (since an indirect transfer leads to interaction), for an absence of a rate-limiting isomerization of the enzyme and for an absence of certain types of interaction between active centres (Britton, 1965a, 1966b, 1967b). It might have been desirable to make observations at lower substrate concentrations where the enzyme was only partially saturated. Nevertheless, the present results, especially when account is taken of the lack of non-competitive product inhibition and the fact that the enzyme has only one active centre (Ray \& Roscelli, 1964b), must provide strong evidence for the direct transfer of glucose residues whatever mechanism may be involved.

At the highest concentrations (Fig. 3) countertransport of ${ }^{14} \mathrm{C}$-labelled substrates occurred. As discussed above, this result constitutes strong evidence that the flux ratio departs from the Independence relationship (eqn. 21) in the direction of exchange diffusion (Britton, 1965a, 1966b). It follows that there must be a method of thermodynamically coupling the energy that is made 
available when the non-radioactive G1P is converted into $\mathrm{G} 6 \mathrm{P}$ in the induced-transport test to the conversion of radioactive G6P into G1P, since this latter conversion occurs against the energy gradient. Two possible mechanisms have been proposed (Britton, 1965a, 1966b, 1967b). In one there is a rate-limiting isomerization of the free enzyme. During the reaction the two isomeric forms are not at equilibrium and the displacement of this step from equilibrium mediates the transfer of energy. This is comparable with the mobile-carrier mechanism that has been used to explain exchange diffusion in the permeability field. The other mechanism involves an interaction between active centres in a multivalent enzyme. This was discussed by Britton $(1967 b)$, but to explain the interaction some form of isomeric change in the enzyme again seems to be required. Since rabbit phosphoglucomutase has only one active centre/molecule (Ray \& Roscelli, $1964 b$ ) it would appear that the latter possibility can be excluded, and counter-transport must constitute good evidence for an isomerizing step involving the free enzyme. The assumptions involved in deriving a numerical value for the rate constant are discussed in the Appendix. However, the true value of the rate constant may be expected to be about one-sixth of the apparent rate constant $\left(4.5 \times 10^{7} \mathrm{sec}^{-1}\right)$ because of the low activity coefficients of the substrates at high concentrations. There is an additional uncertainty because of the need to assume that the activity coefficients of the different enzyme species are equal.

(c) Significance of the induced-transport tests with 32P-labelled substrates. As discussed above, the induced transport of $32 \mathrm{P}$-labelled substrates constitutes strong evidence that the flux ratio for phosphate deviates from the Independence relationship (eqn. 21) in the direction of the long-pore effect. It follows that in the induced-transport test radioactive phosphate from the G1P is transferred to the G6P against the energy gradient (Britton, 1965a), and it is necessary for the reaction to contain a step that will transfer the energy made available from the conversion of non-radioactive G1P into G6P to the radioactive species. The indirect transfer of phosphate provides such a step, since when the ${ }^{32} \mathrm{P}$-labelled phosphate is attached to the enzyme (or to an enzyme-G1P6P complex) the subsequent fate of the 32P-labelled phosphate depends on whether a molecule of G1P or G6P reacts next with the enzyme, and it is immaterial whether this molecule is labelled or not. Interaction of this type is very similar to that proposed by Hodgkin \& Keynes (1955) in their long-pore model to explain $\mathrm{K}^{+}$permeability of the squid axon. Alternatively, interaction could occur if the enzyme were multivalent and there were interaction between active centres (Britton, 1965a, 1966b, 1967b). The type of interaction that would be required would be similar to that suggested to occur in multivalent carriers (Hodgkin \& Keynes, 1955; Britton, 1965b). However, in the present case the enzyme has only one active centre/molecule (Ray \& Roscelli, 1964b), and the results obtained with ${ }^{14} \mathrm{C}$-labelled substrates are incompatible with such interactions since the glucose flux ratio should be similarly influenced. Thus the indirect transfer of phosphate appears to provide the only possible explanation of the experimental results.

Since the phosphate flux ratio was equal to the square of the right-hand side of the Independence relationship (eqn. 21) over a considerable concentration range, it can further be argued that there must be only one intermediate phosphate in the reaction whatever the mechanism. Thermodynamic considerations (Britton, 1966a) indicate an average of at least one intermediate phosphate, but a larger number of intermediate phosphates would give rise to a greater degree of interaction, as is seen when the enzyme is considered as a molecular shuttle (Britton, 1966b). Although it would be possible for interactions produced by different steps in the reaction to cancel each other, the degree of cancellation would be expected to depend on the substrate concentrations, and the fact that the phosphate flux ratio was independent of these concentrations constitutes strong evidence for a single intermediate phosphate.

Ratio of glucose flux to phosphate flux at chemical equilibrium. The ratio of the fluxes at chemical equilibrium will not be influenced by non-ideal behaviour of the substrates except insofar as there may be a rate-limiting isomerization of the enzyme, when the numerical value of $\alpha$ (see eqns. 10 and 20) will be affected. On the other hand the flux ratio will be sensitive to any isotope effects. The fact that the ratio of the fluxes was $2: 1$ over a $2 \frac{1}{2}$-fold concentration range is compatible with the direct transfer of glucose residues and the indirect transfer of phosphate with a single intermediate phosphate, and with an isomerization of the free phosphoenzyme that is not substantially rate-limiting at the substrate concentrations used. There are, however, other mechanisms that would yield the same result, though they may appear to be unlikely. Thus the glucose residue might be transferred indirectly with one intermediate glucose and the phosphate might be transferred via three intermediate phosphates. Correspondingly, there might be two intermediate glucose residues and five intermediate phosphate groups. Unlike the induced-transport tests, interaction between active centres will not influence the flux ratio at equilibrium.

It is seen from eqn. (10) that the flux ratio should increase at high substrate concentrations if there is a slow isomerization of the free enzyme. However, 
calculation shows that this is a far less sensitive test for such a step than the induced-transport test with ${ }^{14} \mathrm{C}$-labelled substrates. Thus a value of $\alpha$ that provides a $10 \%$ rise in the radioactivity in the G1P in the induced-transport test will produce only an $0.8 \%$ change in the ratio of the glucose flux to the phosphate flux when equilibrium has been reached.

General conclusions. To summarize, the inducedtransport tests and the flux ratio at equilibrium provide very strong evidence for the reaction proceeding entirely by the direct transfer of glucose from product to reactant and the indirect transfer of phosphate with one intermediate phosphate. These conclusions are independent of the detailed reaction mechanism and require no assumptions about the constancy of the rate constants. Further, strong evidence is obtained for an isomerization of the free phosphoenzyme with an apparent rate constant of about $4.5 \times 10^{7} \mathrm{sec}^{-1}$. The true rate constant would appear to be about one-sixth of this value. Of the three models proposed in the introduction it is clear that mechanism 1 represents the only possible mechanism. It is also clear that the isomerization of the phosphoenzyme is so rapid that it can only involve a small chemical change. This might involve a rearrangement of hydrogenbonding of the phosphate by histidine.

It has been reported that rabbit muscle phosphoglucomutase contains two isoenzymes (Joshi et al. 1967). However, the conclusions drawn about the mechanism of the reaction are unaffected, since these do not depend on the rate constants. Some uncertainty must be introduced about the value of the rate constant for the isomerization, since one isoenzyme might possess a higher rate of isomerization than the other, but conclusions about the order of magnitude of this rate constant will still be valid.

Ray \& Roscelli (1964b) found no evidence for isomerization of the phosphoenzyme when they examined the reaction for product inhibition, and they concluded that the apparent rate constant for the isomerization step, if it should exist, must be greater than $10^{7} \mathrm{sec}^{-1}$. Similar concentrations of substrates were used as in the induced-transport tests with ${ }^{14} \mathrm{C}$-labelled substrates, and it therefore appears that induced transport is a more sensitive test for isomerization of the enzyme. Further, the induced-transport test does not require the assumption that the rate constants are unaffected by the presence of substrate. Both tests, however, will be similarly influenced by non-ideal behaviour of the solutions.

Although in the above the induced-transport tests and the flux ratio at equilibrium have been discussed particularly in relation to the phosphoglucomutase reaction, it is clear that such tests should be generally applicable to enzyme studies. The relative freedom of induced-transport tests from isotope effects and the relatively unambiguous interpretation of the results should make them particularly useful as kinetic tools.

We thank the Wellcome Trust for financial support. J.B.C. is a Wellcome Trust Research Assistant.

\section{REFERENCES}

Bartlett, G. R. (1959). J. biol. Chem. 234, 466. Britton, H. G. (1963). Nature, Lond., 198, 190. Britton, H. G. (1965a). Nature, Lond., 205, 1323. Britton, H. G. (1965b). J. theoret. Biol. 10, 28. Britton, H. G. (1966a). Nature, Lond., 209, 296. Britton, H. G. (1966b). Arch. Biochem. Biophys. 117, 167. Britton, H. G. (1967a). Biochem. J. 103, 7 P.

Britton, H. G. (1967b). Arch. Biochem. Biophys. 121, 522. Brody, S. \& Tatum, E. L. (1967). Proc. nat. Acad. Sci., Wash., 58, 923.

Cascales, M. \& Grisolia, S. (1966). Biochemistry, 5, 3116.

Colowick, S. P. \& Sutherland, E. W. (1942). J. biol. Chem. $144,423$.

Cori, C. F., Colowick, S. P. \& Cori, G. T. (1937). J.biol. Chem. $121,465$.

Cori, C. F., Cori, G. T. \& Green, A. A. (1943). J. biol. Chem. $151,39$.

Hanabusa, K., Dougherty, H. W., del Río, C., Hashimoto, T. \& Handler, P. (1966). J. biol. Chem. 241, 3930.

Hashimoto, T. \& Handler, P. (1966). J. biol. Chem. 241, 3940.

Hodgkin, A. L. \& Keynes, R. D. (1955). J. Physiol. 128, 61. Joshi, J. G., Hooper, J., Kuwaki, T., Sakurada, T., Swanson, J. R. \& Handler, P. (1967). Fed. Proc. 26, 557.

Mortimer, D. C. (1952). Canad. J. Chem. 30, 653.

Najjar, V. A. (1948). J. biol. Chem. 175, 281.

Najjar, V. A. \& Pullman, M. E. (1954). Science, 119, 631.

Nelson, N. (1944). J. biol. Chem. 153, 375.

Ray, W. J., jun. \& Roscelli, G. A. (1964a). J. biol. Chem. 239, 1338.

Ray, W. J., jun. \& Roscelli, G. A. (1964b). J. biol. Chem. 239, 3935.

Ray, W. J., jun. \& Roscelli, G. A. (1966a). J. biol. Chem. 241, 2596.

Ray, W. J., jun. \& Roscelli, G. A. (1966b). J. biol. Chem. 241, 3499.

Ray, W. J., jun., Roscelli, G. A. \& Kirkpatrick, D. S. (1966). J. biol. Chem. 241, 2603.

Somogyi, M. (1945). J. biol. Chem. 160, 62.

Sugino, Y. \& Miyoshi, Y. (1964). J. biol. Chem. 239, 2360. 


\section{APPENDIX}

Part I : conditions for induced transport

Consider a chemical reaction:

$$
\mathbf{A} \rightleftharpoons \mathbf{B}
$$

and suppose that both labelled and unlabelled reactants are present. Then at equilibrium:

$$
\frac{f_{\mathrm{A}}^{0}[\mathrm{~A}]_{0}}{f_{\mathrm{B}}^{0}[\mathrm{~B}]_{0}}=K
$$

and

$$
\frac{f_{\mathrm{A}}^{0}\left[\mathrm{~A}^{*}\right]_{0}}{f_{\mathrm{B}^{*}}^{0}\left[\mathrm{~B}^{*}\right]_{0}}=K^{*}
$$

where $[\mathrm{A}]_{0}$ and $[\mathrm{B}]_{0}$ are the concentrations, $f_{\mathrm{A}}^{0}$ and $f_{\mathrm{B}}^{0}$ are the activity coefficients and $K$ is the equilibrium constant for the unlabelled species, and $\left[\mathrm{A}^{*}\right]_{0},\left[\mathrm{~B}^{*}\right]_{0}$ etc. have the same significance for the labelled species.

Let:

$$
\begin{aligned}
& m_{\mathrm{A} \rightarrow \mathrm{B}}=\text { flux of unlabelled } \mathrm{A} \text { to } \mathrm{B} \\
& m_{\mathrm{B} \rightarrow \mathrm{A}}=\text { flux of unlabelled } \mathrm{B} \text { to } \mathrm{A}
\end{aligned}
$$

Then:

$$
\begin{aligned}
& \text { Flux of labelled A to } \mathrm{B}=p_{0} m_{\mathrm{A} \rightarrow \mathrm{B}} \frac{f_{\mathrm{A}^{*}}^{0}\left[\mathrm{~A}^{*}\right]_{0}}{f_{\mathrm{A}}^{0}[\mathrm{~A}]_{0}} \\
& \text { Flux of labelled } \mathrm{B} \text { to } \mathrm{A}=q_{0} m_{\mathrm{B} \rightarrow \mathrm{A}} \frac{f_{\mathrm{B}^{*}}^{0}\left[\mathrm{~B}^{*}\right]_{0}}{f_{\mathrm{B}}^{0}[\mathrm{~B}]_{0}}
\end{aligned}
$$

where $p_{0}$ and $q_{0}$ are factors to allow for isotope effects at chemical equilibrium.

Since there is equilibrium:

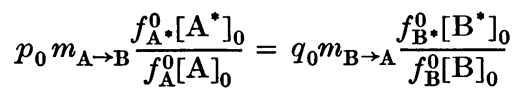

From eqns. (1), (2) and (5):

$$
\frac{p_{0}}{q_{0}}=\frac{K}{K^{*}}
$$

Suppose that unlabelled $\mathbf{A}$ is added to the equilibrium mixture. Then:

$$
\begin{aligned}
& \text { Flux of labelled A to } \mathrm{B}=p m_{\mathrm{A} \rightarrow \mathrm{B}} \frac{f_{\mathrm{A}^{*}}\left[\mathrm{~A}^{*}\right]_{0}}{f_{\mathrm{A}}[\mathrm{A}]} \\
& \text { Flux of labelled } \mathrm{B} \text { to } \mathrm{A}=q m_{\mathrm{B} \rightarrow \mathrm{A}} \frac{f_{\mathrm{B}^{*}}\left[\mathrm{~B}^{*}\right]_{0}}{f_{\mathrm{B}}[\mathrm{B}]_{0}}
\end{aligned}
$$

where $f_{\mathrm{A}}, f_{\mathrm{B}}$ etc. are activity coefficients and $p$ and $q$ are isotope factors for the particular non-equilibrium conditions.

Let:

$$
\frac{p / q}{p_{0} / q_{0}}=n
$$

Co-transport will occur if the flux of labelled A to $B$ exceeds the flux of labelled $B$ to $A$, and countertransport will take place if the converse should hold. If the two fluxes are equal no induced transport of the labelled species will occur. Thus from eqns. (2), (6), (7) and (8):

$$
n \frac{m_{\mathrm{A} \rightarrow \mathrm{B}}}{m_{\mathrm{B} \rightarrow \mathrm{A}}}=\frac{f_{\mathrm{A}}[\mathrm{A}] f_{\mathrm{B}^{*}} f_{\mathrm{A}^{*}}^{0}}{f_{\mathrm{B}}[\mathrm{B}]_{0} K f_{\mathbf{A}^{*}} f_{\mathbf{B}^{*}}^{0}}
$$

for no net transport of labelled material;

$$
n \frac{m_{\mathbf{A} \rightarrow \mathbf{B}}}{m_{\mathbf{B} \rightarrow \mathbf{A}}}>\frac{f_{\mathbf{A}}[\mathbf{A}] f_{\mathbf{B}^{*}} f_{\mathbf{A}^{*}}^{0}}{f_{\mathbf{B}}[\mathbf{B}]_{0} K f_{\mathbf{A}^{*}} f_{\mathbf{B}^{*}}^{0}}
$$

for co-transport of labelled material;

$$
n \frac{m_{\mathrm{A} \rightarrow \mathbf{B}}}{m_{\mathrm{B} \rightarrow \mathbf{A}}}<\frac{f_{\mathrm{A}}[\mathrm{A}] f_{\mathbf{B}^{*}} f_{\mathbf{A}^{*}}^{0}}{f_{\mathbf{B}}[\mathrm{B}]_{0} K f_{\mathbf{A}^{*}} f_{\mathbf{B}^{*}}^{0}}
$$

for counter-transport of labelled material.

In eqns. (10), (11) and (12) $m_{\mathrm{A} \rightarrow \mathrm{B}}$ and $m_{\mathrm{B} \rightarrow \mathrm{A}}$ are the fluxes of unlabelled material. Clearly, however, when the isotope is present in trace quantities only these will approximate to the total fluxes.

Isotope effects. $K^{*}$ does not appear in eqns. (10), (11) and (12), and the extent by which $K^{*}$ differs from $K$ as a result of isotope effects will not influence the criteria for induced transport. The factor $n$ will also be unity even in the presence of isotope effects if the reaction has only one pathway, since the labelled and unlabelled molecules must take part in exactly the same reactions and $p$ and $q$ will always remain in constant proportion. However, $n$ will not necessarily be unity if the reaction has alternative pathways, since the labelled species may follow one pathway preferentially and the labelled species will be influenced by the flux ratio for this particular pathway rather than by the flux ratio for the overall reaction. Nevertheless the occurrence of induced transport may still give rise to the thermodynamic necessity of postulating a mechanism to account for the transfer of energy from the unlabelled to the labelled species (Britton, $1965,1966)$, and may therefore provide evidence for or against certain steps in the reaction. The influence of isotope effects on the activity coefficients is discussed below.

Non-ideal behaviour of solutions. If the ratio $f_{\mathrm{A}^{*}} / f_{\mathrm{B}^{*}}$ is uninfluenced by the addition of nonradioactive $A$, then the activity coefficients for the labelled species cancel out in eqns. (10), (11) and (12), and assuming $n$ is unity the criteria for no induced transport, co-transport and countertransport become that the flux ratio should obey the Independence relationship (eqn. 21 of the main paper), should show the long-pore effect and should 
show exchange diffusion respectively. Isotope effects may be expected to have little influence on activity coefficients, and in most cases it would seem sufficient to demonstrate that the ratio $f_{A} / f_{B}$ is uninfluenced by the addition of $A$ in order to assume that the activity coefficients for the labelled species cancel.

From the above it is seen that it should also be possible to use an alternative substrate rather than labelled material in the induced-transport test.

Although isotope effects may often have no influence on the criteria for the occurrence of induced transport, the magnitude of any induced transport is sensitive to isotope effects, as shown in part II of the Appendix.

Although from general considerations $n$ may be expected to be unity if there is a single pathway, the following argument, which may be employed when the isotope is present in trace quantities, may be useful.

Consider, for example, the transport of ${ }^{32} \mathrm{P}$ labelled phosphate groups from G1P to G6P as is shown for mechanism 1 in Scheme 1 of the main paper, and let the rate constants for the reactions involving ${ }^{32} \mathrm{P}$-labelled phosphate (as shown in Scheme 1 of the main paper) be $k_{+1}^{\prime}, k_{+2}^{\prime}$ etc. and the rate constants for the non-isotopic reactions be $k_{+1}, k_{+2}$ etc. Since ${ }^{32} \mathrm{P}$-labelled phosphate groups are present in trace quantities only, the possibility that two molecules of [ $\left.{ }^{32} \mathrm{P}\right] \mathrm{G} 1 \mathrm{P}$ react successively with the enzyme can be ignored, so that the sequence shown will be the only one for the transfer of ${ }^{32} \mathrm{P}$ labelled phosphate from G1P to G6P.

The overall flux ratio is the product of the flux ratio for the individual steps (Britton, 1963) and it is possible to write:

Flux of [ $\left.{ }^{32} \mathrm{P}\right]$ phosphate from G1P to G6P

Flux of [32P]phosphate from G6P to G1P

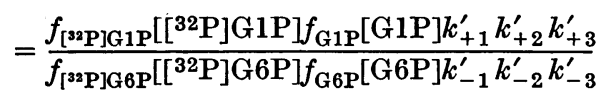

and

Flux of unlabelled phosphate from G1P to G6P

Flux of unlabelled phosphate from G6P to G1P

$$
=\frac{f_{\mathrm{G} 1 \mathrm{P}}{ }^{2}[\mathrm{G} 1 \mathrm{P}]^{2} k_{+1} k_{+2} k_{+3}}{f_{\mathrm{G} 6 \mathrm{P}^{2}}[\mathrm{G} 6 \mathrm{P}]^{2} k_{-1} k_{-2} k_{-3}}
$$

where [G1P], [G6P] etc. represent concentrations and $f_{\mathrm{G} 1 \mathrm{P}}$ etc. activities.

Let $\mathrm{G} 1 \mathrm{P}=\mathrm{A}$ and $\mathrm{G} 6 \mathrm{P}=\mathrm{B}$; then by comparisons with eqns. (7) and (8):

$$
\frac{p}{q}=\frac{k_{+1}^{\prime} k_{+2}^{\prime} k_{+3}^{\prime} k_{-1} k_{-2} k_{-3}}{k_{-1}^{\prime} k_{-2}^{\prime} k_{-3}^{\prime} k_{+1} k_{+2} k_{+3}}=\frac{K}{K^{*}}
$$

Thus by comparison with eqn. (6) $n$ will be unity.

\section{Part II}

Consider a chemical reaction:

$$
\mathrm{A} \rightleftharpoons \mathrm{B}
$$

Net rate of accumulation of isotopically labelled A is given by:

$$
\begin{aligned}
V \frac{\mathrm{d}\left[\mathrm{A}^{*}\right]}{\mathrm{d} t}= & q^{\prime} \frac{\left[\mathrm{B}^{*}\right]}{[\mathrm{B}]}(\text { flux of } \mathrm{B} \text { to } \mathrm{A}) \\
& -p^{\prime} \frac{\left[\mathrm{A}^{*}\right]}{[\mathrm{A}]}(\text { flux of } \mathrm{A} \text { to } \mathrm{B})
\end{aligned}
$$

where $[\mathrm{A}]$ and $[\mathrm{B}]$ represent the total concentrations and $\left[\mathrm{A}^{*}\right]$ and $\left[\mathrm{B}^{*}\right]$ the concentrations of labelled $A$ and $\mathrm{B}, t$ is time, $p^{\prime}$ and $q^{\prime}$ are factors to allow for isotope effects and $V$ is volume of the solution.

Let:

$$
\frac{\text { Flux of } \mathrm{A} \text { to } \mathrm{B}}{\text { Flux of } \mathrm{B} \text { to } \mathrm{A}}=r
$$

Then since:

Net flux $=($ flux of A to B $)-($ flux of B to A)

$$
\text { Flux of } A \text { to } B=\frac{r}{r-1} \text { (net flux of A to B) }
$$

and

$$
\text { Flux of B to } A=\frac{1}{r-1} \text { (net flux of A to B) }
$$

Also

$$
\text { Net flux of } \mathbf{A} \text { to } \mathbf{B}=-V \frac{\mathrm{d}[\mathrm{A}]}{\mathrm{d} t}
$$

Substituting eqns. (18), (19) and (20) into eqn. (17):

$$
\frac{\mathrm{d}\left[\mathrm{A}^{*}\right]}{\mathrm{d} t}=-\frac{\mathrm{d}[\mathrm{A}]}{\mathrm{d} t}\left(q^{\prime} \frac{\left[\mathrm{B}^{*}\right]}{[\mathrm{B}](r-1)}-p^{\prime} \frac{\left[\mathrm{A}^{*}\right] r}{[\mathrm{~A}](r-1)}\right)
$$

If $p^{\prime}=q^{\prime}=1$ (i.e. no isotopic effects are present):

$$
\frac{\mathrm{d}\left[\mathrm{A}^{*}\right]}{\mathrm{d}[\mathrm{A}]}=\frac{\left[\mathrm{A}^{*}\right] r}{[\mathrm{~A}](r-1)}-\frac{\left[\mathrm{B}^{*}\right]}{[\mathrm{B}](r-1)}
$$

It is also possible to write:

$$
[\mathrm{A}]+[\mathrm{B}]=T
$$

and

$$
\left[\mathrm{A}^{*}\right]+[\mathrm{B} *]=N
$$

where $T$ is total concentration of substrate and $N$ is the total concentration of labelled substrate. Eqn. (24) assumes that the amount of label associated with the cofactor is negligible or constant.

The following relationships for $r$ were introduced into eqn. (22) derived from the flux-ratio equations 
given in the Theory section of the main paper $([\mathrm{A}]=[\mathrm{G} 1 \mathrm{P}],[\mathrm{B}]=[\mathrm{G} 6 \mathrm{P}])$ :

$$
r=\frac{[\mathrm{A}](1+\alpha K[\mathrm{~B}])}{K[\mathrm{~B}](1+\alpha[\mathrm{A}])}
$$

derived from eqn. (9) of the main paper.

$$
r=\frac{[\mathrm{A}]^{2}}{K^{2}[\mathrm{~B}]^{2}}
$$

derived from eqn. (8) or eqn. (19) of the main paper assuming :

$$
\frac{1+\alpha K[\mathrm{~B}]}{1+\alpha[\mathrm{A}]}=1
$$

This simplification was adopted in view of the numerical value of $\alpha$ and the low concentrations of G1P and G6P employed in the experiments.

$$
r=\frac{[\mathrm{A}]^{3}}{K^{3}[\mathrm{~B}]^{3}}
$$

derived from eqn. (18) of the main paper by making the same assumption as in (b) above.

$$
r=\frac{[\mathrm{A}]^{2}}{(1-p) K^{2}[\mathrm{~B}]^{2}+p K[\mathrm{~A}][\mathrm{B}]}
$$

derived by assuming that a fraction $(1-p)$ of the flux $\mathrm{A}$ to $\mathrm{B}$ was associated with a flux of $\mathrm{B}$ to $\mathrm{A}$ given by the value of $r$ in eqn. (26) and that a fraction $(p)$ of the flux of A to B was associated with a flux of $B$ to $A$ given by eqn. (25), assuming:

$$
\frac{1+\alpha K[\mathrm{~B}]}{1+\alpha[\mathrm{A}]}=1
$$

as in (b) above.

$$
\text { (e) } \quad r=\frac{[\mathrm{A}]^{3}}{(1-p) K^{2}[\mathrm{~A}][\mathrm{B}]^{2}+p K^{3}[\mathrm{~B}]^{3}}
$$

derived by assuming that a fraction $(1-p)$ of the flux of A to B was associated with a flux of B to A given by eqn. (26) and that a fraction $(p)$ of the flux of A to B was associated with a flux of B to A given by eqn. (27).

$[\mathrm{B}]$ and $\left[\mathrm{B}^{*}\right]$ were eliminated from eqn. (22) by using eqns. (23) and (24) and the resulting equation was integrated numerically with an ICL 1900 series computer from the initial conditions given in the legend to Fig. 1. It is seen that $\alpha$ was closely proportional to the magnitude of the countertransport over the range calculated.

If the solutions show non-ideal behaviour activity coefficients should be appropriately introduced into the expressions for $r$. However, when the activity

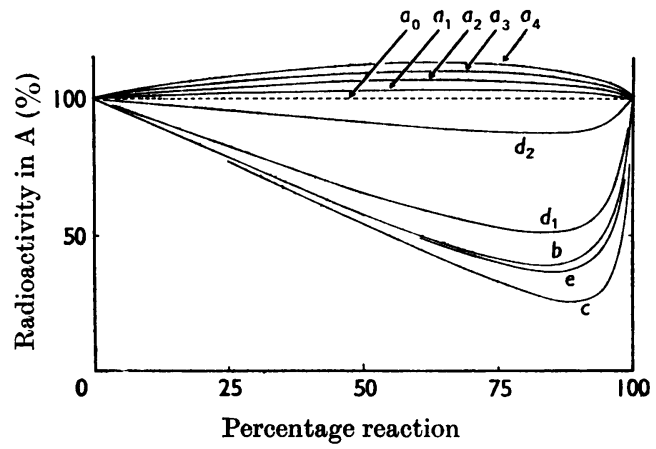

Fig. 1. Theoretical induced-transport curves obtained by integrating eqn. (22). The letters $a, b, c$ etc. refer to the expressions for $r$ that were substituted into eqn. (22). For curve $a_{0}, \alpha=0$; for curves $a_{1}, a_{2}, a_{3}$ and $a_{4}, \alpha[\mathrm{A}]_{1}$ was $0 \cdot 1$, $0.2,0.3$ and 0.4 respectively, where $[A]_{\mathrm{I}}$ represents the initial value of $A$. For curve $d_{1}, p=0.8$; for curve $d_{2}$, $p=0 \cdot 2$; for curve $e, p=0 \cdot 2$. Initial values: for curves $a_{0}-a_{4}$, $[\mathrm{A}]_{\mathrm{I}}=47 \cdot 14,[\mathrm{~B}]_{\mathrm{I}}=39 \cdot 2$; for curves $b, c, d_{1}, d_{2}$ and $e$, $[\mathrm{A}]_{\mathrm{I}}=1.044,[\mathrm{~B}]_{\mathrm{I}}=0.630 . \quad K=1 / 17 \cdot 2$. If $\alpha[\mathrm{A}]_{\mathrm{I}}=0.3$, $\alpha=0.637 \times 10^{-2}$.

coefficients for $\mathrm{A}$ and $\mathrm{B}$ are equal, as in the present case, the coefficients cancel in eqns. (26)-(29), but the numerical value of $\alpha$ in eqn. (25) is changed (see part IV of the Appendix).

In eqns. (28) and (29) for simplicity it has been arbitrarily assumed that the fluxes of $\mathrm{A}$ to $\mathrm{B}$ through the different pathways remain in the same proportion throughout the reaction. If two enzymes are involved, for example, it may be more realistic to assume that the net fluxes remain in constant proportion. A consideration of the relationship between the fluxes (eqn. 18), however, shows that the induced. transport curves will remain of the same form for the initial part of the reaction and that the differences in the later parts of the curves will be relatively small.

\section{Part III : calculation of mean ionic activity coefficients for $G 1 P$ and $G 6 P$}

The mean ionic activity at the lowest concen. tration at which measurements were made $(11.78 \mathrm{~mm})$ in Fig. 6 of the main paper was calculated from the simplified Debye-Hückel relationship:

$$
f_{ \pm}=\frac{0.580 \times 2 \times \sqrt{ } s}{1+\sqrt{ } 8}
$$

where $f_{ \pm}$is the mean ionic activity at $0^{\circ}$ and $s$ is the ionic strength. The change in activity coefficient with increasing concentration was then calculated from the freezing-point-depression curve by using standard techniques (see Moore, 1957). 
Part IV: calculation of the rate constants for the isomerization of the phosphoenzyme

From eqns. (3) and (5) in the main paper in the presence of excess of G1P6P :

$$
K_{\mathrm{G} 1 \mathrm{P}}=\frac{\left(k_{+3}+k_{-3}\right)\left(k_{-1}+k_{+2}\right)}{k_{+1}\left(k_{+2}+k_{+3}\right)}
$$

where $K_{\mathrm{G} 1 \mathrm{P}}$ is the apparent Michaelis constant for G1P in the presence of excess of G1P6P, and

$$
\frac{V_{\max }}{[\mathrm{E}]}=\frac{k_{+2} k_{+3}}{k_{+2}+k_{+3}}
$$

where $V_{\max }$ is the maximum initial velocity with G1P as substrate in the presence of excess of G1P6P.

From the expression for $\alpha$ (eqn. 11 of the main paper) it follows that:

$$
\frac{\alpha K_{\mathrm{G} 1 \mathrm{P}}[\mathrm{E}]}{V_{\max .}}=\frac{1}{k_{+3}}+\frac{1}{k_{-3}}
$$

From the present work $\alpha=0.637 \times 10^{-2} \mathrm{~mm}^{-1}$ and, from Ray \& Roscelli (1966), $K_{\mathrm{G} 1 \mathrm{P}}=5 \cdot 2 \mu \mathrm{M}$ and $V_{\max } . /[\mathrm{E}]=750$ sec. $^{-1}$. Thus:

$$
\frac{1}{k_{+3}}+\frac{1}{k_{-3}}=\frac{0.637 \times 10^{-2} \times 5.2 \times 10^{-3}}{750}
$$

and if $k_{+3}=k_{-3}$ :

$$
k_{+3}=k_{-3}=4.5 \times 10^{7} \text { sec. }^{-1}
$$

This value of $k_{+3}$ represents an apparent value, since the activity coefficients for G1P and G6P and for the enzyme species have been assumed to be unity.

Taking into account the activity coefficients of G1P and G6P and assuming them to be equal (see the Discussion section of the main paper and part III of the Appendix):

$$
k_{+3}=k_{-3}=4.5 \times 10^{7} \times \frac{f_{\mathrm{G} 1 \mathrm{P}}^{\prime}}{f_{\mathrm{G} 1 \mathrm{P}}^{\prime \prime}}
$$

where $f_{\text {G1P }}^{\prime}$ is the activity coefficient of G1P under the conditions of the induced-transport test and $f_{\text {G1P }}^{\prime \prime}$ is the activity coefficient of G1P under the conditions under which $K_{\mathrm{G} 1 \mathrm{P}}$ was determined.

The ionic strength in the experiments shown in Fig. 3 of the main paper from which $\alpha$ was determined was calculated to be 0.517 , and from Fig. 6 of the main paper the mean ionic activity coefficient for GlP was found to be $\mathbf{0 . 4 1 7}$. The ionic strength in the experiments of Ray \& Roscelli (1966) in which $K_{\mathrm{G} 1 \mathrm{P}}$ was determined was calculated to be 0.1145 and the corresponding mean ionic activity of G1P was found to be 0.792 . If the activity coefficients for $\mathrm{Na}^{+}$are assumed to be 0.79 and 0.84 at the higher and lower ionic strengths it follows that:

$$
\frac{f_{\mathrm{G} 1 \mathrm{P}}^{\prime}}{f_{\mathrm{G} 1 \mathrm{P}}^{\prime \prime}} \bumpeq \frac{1}{6}
$$

'Thus the true rate constant will be about onesixth of the apparent value. Clearly, however, such a calculation can only be regarded as approximate and is subject to the uncertainty involved in the determination of individual ionic activities.

The activity coefficients for the different enzyme species should be introduced in relation to the appropriate rate constants in eqns. (30), (31) and (32), but these activity coefficients cancel if they are equal. The latter will not necessarily be true if the electrical charge on the enzyme molecule is altered by the addition of substrate and this introduces an additional uncertainty into the calculation of the true rate constant. However, it would seem unlikely that such factors would alter the order of magnitude of the rate constants.

We thank Mr G. F. Parker (British Petroleum Co. Ltd., London, E.C. 2) for writing the computer programme and integrating eqn. (22) in Part II of the Appendix.

\section{REFERENCES}

Britton, H. G. (1963). J. Physiol. 170, 1.

Britton, H. G. (1G.5). Nature, Lond., 205, 1323.

Britton, H. G. (19tif). Nature, Lond., 209, 296.

Moore, W. J. (1957). Physical Chemistry, pp. 455-456. London: Longmans, Green and Co. Ltd.

Ray, W. J., jun. \& Roscelli, G. A. (1966). J.biol.Chem. 241, 2596. 